\title{
The difference in poststimulus suppression between residual inhibition and forward masking
}

P.H. Bourez, P. Fournier, and A. Noreña* Centre National de la Recherche Scientifique, Aix-Marseille University, Marseille, France

*Corresponding author:Tel.: +33-4-13550865, e-mail address: arnaud.norena@univ-amu.fr

\begin{abstract}
The phenomenon of tinnitus masking (TM) and residual inhibition (RI) of tinnitus are two ways to investigate how external sounds interact with tinnitus: TM provides insight on the fusion between external sound activity and tinnitus related activity while RI provides insight on how the external sound might suppress the tinnitus related activity for a period of time. Differences in masking level between the tinnitus and an external tone with tinnitus characteristics (frequency, loudness) have previously shown a high level of heterogeneity. The difference in poststimulus suppression between the two, that is, residual inhibition for the former, and forward masking for the latter, has never been explored. This study aims to investigate minimum masking levels (MMLs) and minimum residual inhibition levels (MRILs) of tinnitus and of an external tone mimicking tinnitus while using diotic and dichotic noises. Pulsed narrowband noises ( 1 octave width and centered at $1 \mathrm{kHz}$, frequency of the hearing loss slope, tinnitus frequency) and white noise were randomly presented to 20 tinnitus participants and 20 controls with an external tone mimicking tinnitus $(4 \mathrm{kHz}$, intensity level corresponding to tinnitus loudness). The MML values obtained for the masking of tinnitus and for the mimicking external sounds were very similar. On the other hand, the MRILs were significantly different between the tinnitus and the mimicking external sounds within tinnitus participants. They were also different between the tinnitus participants and the controls. Overall, for both within and between comparisons, the MRIL values were much higher to produce a poststimulus suppression for the mimicking sound than for the tinnitus. The results showed no significant differences between the diotic and dichotic conditions. These results corroborate other findings suggesting that the tinnitus-related neural activity is very different from the stimulus-related neural activity. The consequences of this last finding are discussed.
\end{abstract}




\section{Keywords}

Tinnitus, Tinnitus masking, Residual inhibition, Forward masking, Poststimulus suppression, Diotic, Dichotic, Minimum masking level, Minimum residual inhibition level

\section{Introduction}

Subjective tinnitus is an aberrant sensation of ringing or buzzing in the ears or the head. It is produced by the auditory system in the absence of any external acoustical source (Baguley et al., 2013). This phantom sensation can take various forms resembling everyday sounds (Stouffer and Tyler, 1990) and the experience of tinnitus can be quite displeasing for some (McCormack et al., 2014, 2016). Interestingly enough, others may not feel discomfort at all, which may be the result of habituation (Mckenna, 2004). An individual may locate tinnitus to one ear, or both, within the head or even, occasionally, to an auditory percept that is located outside of the head. It has been reported that many individuals may hear more than one tinnitus sound (Basile et al., 2013; Fournier et al., 2018; Norena et al., 2002; Stouffer and Tyler, 1990).

To this day, the mechanisms responsible for the generation of tinnitus are still unclear. However, some authors suggest that the physiological correlate of tinnitus is occurring before or at the cochlear nerve level, e.g. peripheral tinnitus (Guitton and Dudai, 2007; Guitton and Puel, 2004), while others have hypothesized that tinnitus results from the central plastic changes due to hearing loss (Eggermont and Roberts, 2004; Noreña, 2011; Noreña and Farley, 2013; Roberts and Salvi, 2019; Schaette, 2014; Schaette and McAlpine, 2011; Shore et al., 2016). It is still impossible to differentiate tinnitus subtypes in a single patient. Also, it is not excluded that peripheral and central tinnitus might co-occur in the same individual. Importantly, it is likely that tinnitus as a symptom can result from potentially many different mechanisms. One of the main challenges in tinnitus research is to find some way to categorize or subtype tinnitus patients according to the tinnitus physiopathology (Cederroth et al., 2019; Landgrebe et al., 2010; McFerran et al., 2019).

The field of psychoacoustics has been useful to estimate various tinnitus characteristics such as the pitch and loudness of the tinnitus, and the way external sounds can interfere with tinnitus, i.e. its maskability and residual inhibition, the latter being a transient suppression of tinnitus following a brief loud acoustic stimulation. Beyond the simple description of tinnitus perception it is believed that the psychoacoustic characteristics of tinnitus can provide some insights into the mechanisms of tinnitus and potentially help sub-typing patients (Fournier et al., 2018, 2019). As early as in the 1940s and 1950s, Fowler (Fowler, 1940, 1941) and Langenbeck (Langenbeck, 1953) showed that in some cases the tinnitus could be masked easily, while in other cases tinnitus was hardly maskable. It was hypothesized in the former group of patients that tinnitus may originate from the inner ear, while in the latter group tinnitus could result from aberrant activity in the acoustic nerve. 
In addition, psychoacoustic measurements have unveiled some relationship between some tinnitus characteristics and auditory variables such as hearing loss. Indeed, in the cases where tinnitus is accompanied by hearing loss, studies have shown a good correlation between the perceived tinnitus pitch and the frequency band of the hearing loss (Basile et al., 2013; Hébert and Fournier, 2017; Norena et al., 2002; Roberts et al., 2008; Schecklmann et al., 2012; Sereda et al., 2011). Furthermore, the measure of tinnitus "loudness" suggests that the intensity of tinnitus usually resides below $10 \mathrm{~dB}$ SL (Basile et al., 2013; Goodwin and Johnson, 1980; Hallam et al., 1985; Hébert, 2018; Hébert and Fournier, 2017; Mitchell et al., 1993; Moffat et al., 2009; Tyler and Conrad-Armes, 1984).

When researchers have tried to apply some of the best-known psychoacoustic laws that predict our auditory perception to tinnitus, it didn't follow any of these rules. As such, the comparison between tinnitus and external sound perception becomes striking when attempting to "mask" an external tone in comparison to a tinnitus sensation. For example, a study by Penner et al. (1981) showed that after prolonged acoustic stimulation, a noise masker had to be gradually increased in order to continuously mask the tinnitus in 20 participants, while the intensity required to mask an external tone usually remains constant. Indeed, the masking of an external tone is generally associated to a process in which the threshold of a signal is increased by the presence of a masker at the same frequency. This process is made by the mechanical interaction of the former with the latter (Oxenham and Moore, 1994; Plack et al., 2002), whereas tinnitus-masking may only depend on central mechanisms (Feldmann, 1983; Mitchell et al., 1993), as it is suggested for RI (Galazyuk et al., 2017; Roberts, 2007). Furthermore, it has been reported that certain tinnitus subjects have seen their tinnitus masked by tones of almost any frequency (Cazals and Dauman, 1990; Dauman and Cazals, 1989; Feldmann, 1971; Mitchell, 1983; Tyler and Conrad-Armes, 1984), whereas external sounds are much more bounded by frequency selectivity surrounding the signal. Nevertheless, there are cases where tinnitus cannot be masked regardless of the frequency and the intensity of the masker (Burns, 1984; Feldmann, 1971; Fournier et al., 2018; Mitchell, 1983; Tyler and Conrad-Armes, 1984; Vernon and Meikle, 1981) which also is in contradiction with the psychoacoustic laws of external sound perception.

A few well-controlled experiments have been run in order to differentiate tinnitus masking from external sound masking within the same tinnitus participants (Burns, 1984; Fournier et al., 2019; Tyler and Conrad-Armes, 1984). In these studies, different noise maskers were used to compare the level of the masker required to mask tinnitus in comparison to the level of the same maskers required to mask an external sound presented at the same frequency and level as the tinnitus. These studies showed that tinnitus is generally more easily masked than an external sound with the same characteristics (frequency and intensity) when the frequency of the masker is far away from the tinnitus frequency. However, when the masker frequency is centered at the tinnitus frequency, the tinnitus is generally more difficult to mask than the external sound target (Fournier et al., 2019). In addition, noise-like tinnitus was found to be even more difficult to mask than tone-like tinnitus for the frequency 
masker centered at the tinnitus frequency (Fournier et al., 2019). Overall, these studies have shown that the frequency selectivity of tinnitus masking is generally wider than for the masking of an external sound. This suggests that the tinnitus related activity might be generated in the nonlemniscal (nontonotopic) pathway or in a central region where neurons integrate sensory inputs over a wide frequency range (Fournier et al., 2019; Møller, 2007; Møller et al., 1992).

To further extend the knowledge on the mechanisms that might differentiate tinnitus from an external sound perception, we decided to explore the phenomenon of residual inhibition. The main goal of this study was thus to investigate, in a new systematic approach, the difference in poststimulus suppression between tinnitus and an external sound which refers to residual inhibition and forward masking, respectively. Preliminary work on Residual Inhibition (RI), which consists of a temporary suppression of the tinnitus percept after the presentation of a moderate to loud sound stimulation, goes as far back as the early 1970s. Feldmann (1971) found that a large number of subjects experienced a partial or total suppression of their tinnitus lasting from seconds to minutes following the presentation of a relatively intense masker for $30 \mathrm{~s}$ to $1 \mathrm{~min}$. On the other hand, forward masking occurs when a physical sound is suppressed or reduced during a certain period of time following the interruption of a preceding masker. If we present a relatively intense masker sound to a normal hearing listener, and place the onset of a target sound at the offset of the masker, the listener will detect that the onset of the target sound is delayed in time (Meddis and O'Mard, 2005; Oxenham, 2001). The delay will vary in time according to the different psychophysical attributes of the masker and the target sound (Moore, 2012). This well-known psychoacoustic phenomenon is believed to emerge from neural properties conveyed by the preceding masking sound, as neural responses show adaptational properties that prevent the target sound to be decoded before the neurons in the auditory fibers reach resting potential (Harris and Dallos, 1979; Meddis and O'Mard, 2005; Oxenham, 2001). As the psychoacoustic laws of external sound masking and tinnitus masking do not stand on equal ground, our foresight on the mechanisms underlying the inhibition of tinnitus in comparison to those of external sounds is still unclear. These two psychoacoustic phenomena commonly known as residual inhibition for the former, and forward masking for the latter have never been clearly distinguished in a systematic way. This was accomplished by direct comparison of the MRILs of tinnitus, and of an external sound mimicking tinnitus between a tinnitus group and a control group, as well as within a subgroup of tinnitus participants but with two different targets (tinnitus vs external sound mimicking the tinnitus).

Moreover, we decided to insert different phase configurations in our procedure that is dichotic $\left(\mathrm{N}_{\pi} \mathrm{S}_{0}\right)$ and diotic maskers $\left(\mathrm{N}_{0} \mathrm{~S}_{0}\right)$ in order to assess differences in MML and MRIL between them. Indeed, the binaural characteristics of our auditory system have been investigated in the past in order to deliver "tailored" acoustic stimulation to the ears of any given tinnitus patient with some success (Johnson and Hughes, 1992; Searchfield et al., 2016; Tyler and Stouffer, 1991). Basing their approach on Binaural Masking Level Differences (BMLD), it was hypothesized that 
manipulating the interaural phasing of a masking sound in order match the subjective localization of the tinnitus could improve tinnitus masking in subjects. The physiologic origin of binaural unmasking has yet to be discovered, but research suggests the existence of neural units sensitive to interaural variations that may take place at a conjecture level of the auditory nerves coming from the left and right ear. This means that this process could be located somewhere in the brainstem (cochlear nucleus, superior olivary complex), but may also take place in the midbrain (inferior colliculi or adjacent areas), the diencephalon (medial geniculate nucleus) or the cortex. In summary, the process takes place at any central level that is able to integrate binaural inputs, but not at the periphery, this could help differentiate peripheral from central subtype tinnitus.

\section{Methods}

\subsection{Participants}

A study sample of 20 tinnitus participants (mean age: 54 years, ranges 24-74) called the tinnitus group (TG) and 20 control participants (mean age: 27 years, ranges 22-46) without tinnitus called the control group (CG) were included in the study. The study sample demographics are available in Table 1 . A total of 17 tinnitus participants had bilateral tinnitus with seven reporting a louder perception on one side: four predominant in the right ear and three predominant in the left ear. Only three participants reported unilateral tinnitus strictly on the left side. Ten tinnitus participants described their tinnitus as noise-like, seven as tone-like and three reported a mixture of pure tones and noise. The mean hearing thresholds for all standard audiometric frequencies of tinnitus participants and the distribution of the predominant tinnitus pitch are illustrated in Fig. 1. The most prevalent predominant tinnitus pitch was $8 \mathrm{kHz}(n=12)$. Only control participants with normal hearing (screened at $\leq 20 \mathrm{~dB}$ HL across frequencies) were included in the study. In addition, exclusion

Table 1 Sociodemographics of the tinnitus group, the control group and the tinnitus subgroup participants.

\begin{tabular}{l|l|l|l|l|l} 
& $\begin{array}{l}\text { Mean } \\
\text { age in } \\
\text { years (SD) }\end{array}$ & $\begin{array}{l}\text { Sex } \\
\text { (M/F) }\end{array}$ & $\begin{array}{l}\text { Tinnitus } \\
\text { pitch } \\
\text { in Hz (SD) }\end{array}$ & $\begin{array}{l}\text { Tinnitus } \\
\text { duration in } \\
\text { years (SD) }\end{array}$ & $\begin{array}{l}\text { Tinnitus } \\
\text { loudness in } \\
\text { dB SL (SD) }\end{array}$ \\
$\begin{array}{l}\text { Tinnitus group } \\
(n=20)\end{array}$ & $54(14)$ & $14 / 6$ & $7842(1730)$ & $3.84(4)$ & $10.7(5)$ \\
$\begin{array}{l}\text { Control group } \\
(n=20)\end{array}$ & $27(5)$ & $10 / 10$ & - & - & - \\
$\begin{array}{l}P \text {-value } \\
\text { Tinnitus subgroup } \\
(n=10)\end{array}$ & $51.5(14)$ & $7 / 3$ & $7515(1994)$ & $3.54(4)$ & $11.2(6)$ \\
\hline
\end{tabular}

Significant P-value is in bold. 


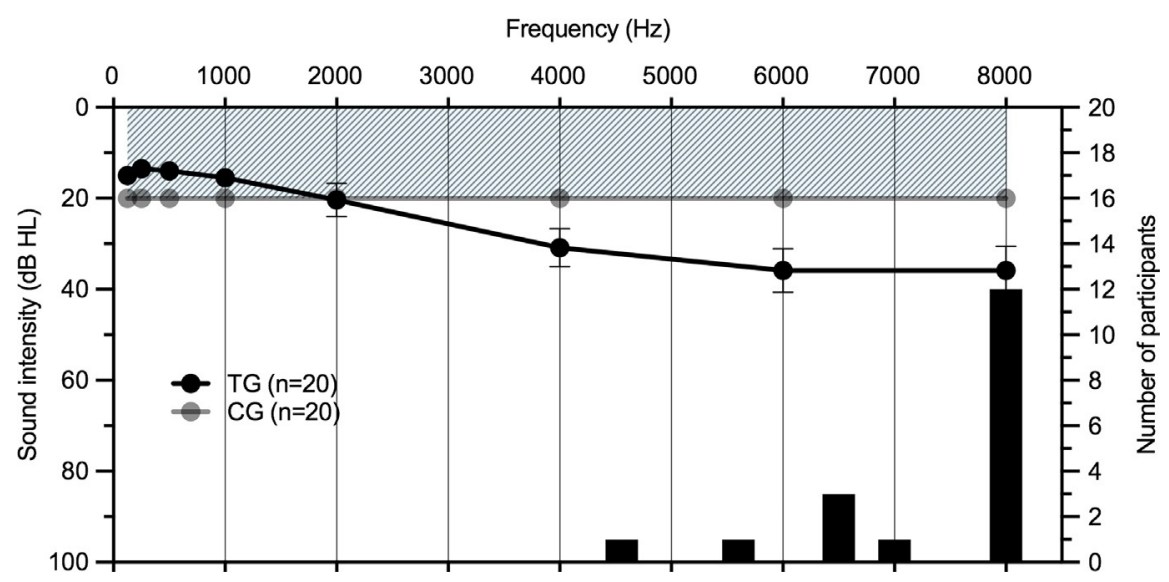

FIG. 1

Mean hearing thresholds of the tinnitus group participants for each of the standard frequencies from 125 to $8000 \mathrm{~Hz}$. The error bars represent the Standard Error of the Mean (SEM). The distribution of the predominant tinnitus pitches across all tinnitus participants is also presented. Two participants are not part of this distribution since the predominant pitch of their tinnitus was above $8000 \mathrm{~Hz}$ (12,000 for both).

criteria for this group included history of tinnitus or any other form of ear-related pathology. For the tinnitus participants, the inclusion criteria included normal hearing thresholds from 0.25 to $8 \mathrm{kHz}$ or symmetrical sensorineural hearing loss. Any tinnitus participant with a binaural difference in hearing thresholds of $>15 \mathrm{~dB}$ HL at two consecutive frequencies $(0.25-8.0 \mathrm{kHz})$ was excluded from the study. This criterion was used to reduce possible bias in binaural processing due to asymmetrical hearing (Hawkins and Wightman, 1980; Jerger et al., 1984). Tinnitus participants were mainly recruited from the IMERTA clinic database (an audiological clinic specialized in tinnitus management based in Marseille, France). Other tinnitus participants were recruited by various means including social media advertisements, poster advertisements placed in clinics and via an ad posted in the "Relais d'information en sciences de la cognition" (https://www.risc.cnrs.fr) website. Most of the control participants were recruited by word of mouth and were mostly students and/or university personnel. All of the participants were informed about the aim of the study and gave written consent (The Comité de Protection des Personnes Nord Ouest IV review board approved this study: reference number: 2018 A01183 52).

\subsection{Audiological assessment measures}

For the tinnitus participants, the hearing thresholds (HT) were assessed monaurally, for both ears, by presenting pure tones from 0.125 to $8 \mathrm{kHz}$ by half-octave steps using the conventional clinical procedures (Harrell, 2002) using Sennheiser HDA-280 connected to an Astera audiometer (Otometrics). For the control participants, a hearing 
screening was performed and consisted in presenting pure tones $(0.125-8 \mathrm{kHz})$ at $20 \mathrm{~dB}$ HL monaurally, in both ears, to the participants and asking whether or not they heard the sound. Control participants passed selection criteria as long as they were able to perceive all the sounds presented.

\subsection{Psychoacoustic measures}

\subsubsection{Equipment}

Psychoacoustic measures were performed using Sennheiser HD-600 supra-auricular headphones connected to a Sound Blaster X-fi HD model SB1240 sound card. All the psychoacoustic measurements were performed in a soundproof room. A MATLAB ${ }^{\circledR}$ program, created in-house, allowed the experimenter to control manually all the stimulus parameters such as the duration, the intensity level, the center frequency, and the bandwidth of any sound presented. The experimenter controlled all the sound presentations from outside the soundproof room and could communicate with the participants at all times via a two-way communication system.

\subsubsection{Tinnitus pitch and loudness matching}

Tinnitus pitch and loudness matching were assessed only for the tinnitus group. They were obtained for each tinnitus patient as follows: The patient was first presented with three generated sounds, that is, white noise, narrowband noise, and pure tones. He was then asked: "which of the three sounds resembles most your tinnitus?". This allowed to determine the type of tinnitus between tone-like, narrowband-like or broadband-like. If the type of tinnitus was tone or narrowband-like, the test continued and we tried to determine precisely the frequency of the tinnitus. To do so, the patient was presented with a sound centered at $4 \mathrm{kHz}$ (NBN or pure tone) and was asked, "is the pitch of the sound higher or lower than your tinnitus?". According to his answer, the experimenter either increased or decreased the pitch of the sound by half of an octave and asked the question again. This continued until the patient reported that the generated sound matched most with his tinnitus. The most prevalent predominant tinnitus pitch was $8 \mathrm{kHz}(n=12)$. Loudness matching was assessed by presenting the previously best pitch-matched sound at a low but audible level (close to HT) and gradually increasing it by $2 \mathrm{~dB}$ steps until the patient reported that the intensity of the sound was similar to the intensity of his tinnitus. The mean loudness matching was of $10.7 \mathrm{~dB}$ SL $(\mathrm{SD}=5.2)$ which is consistent with tinnitus loudness values reported in the literature (Basile et al., 2013; Fournier et al., 2019, 2018; Goodwin and Johnson, 1980; Hébert, 2018; Hébert and Fournier, 2017; Mitchell et al., 1993; Moffat et al., 2009; Tyler and Conrad-Armes, 1984).

\subsubsection{Masker thresholds (MT), minimum masking levels (MMLs) and minimum residual inhibition levels (MRILs)}

The MML and MRIL technique used in the present study was previously developed by Fournier et al. (2018). The stimulus is made of pulsed narrowband noises (1 octave width). However, the pulsed sequence used here differs slightly from the one used in Fournier and collaborators: the ramped continuous stimulus has a fixed-rise time of 


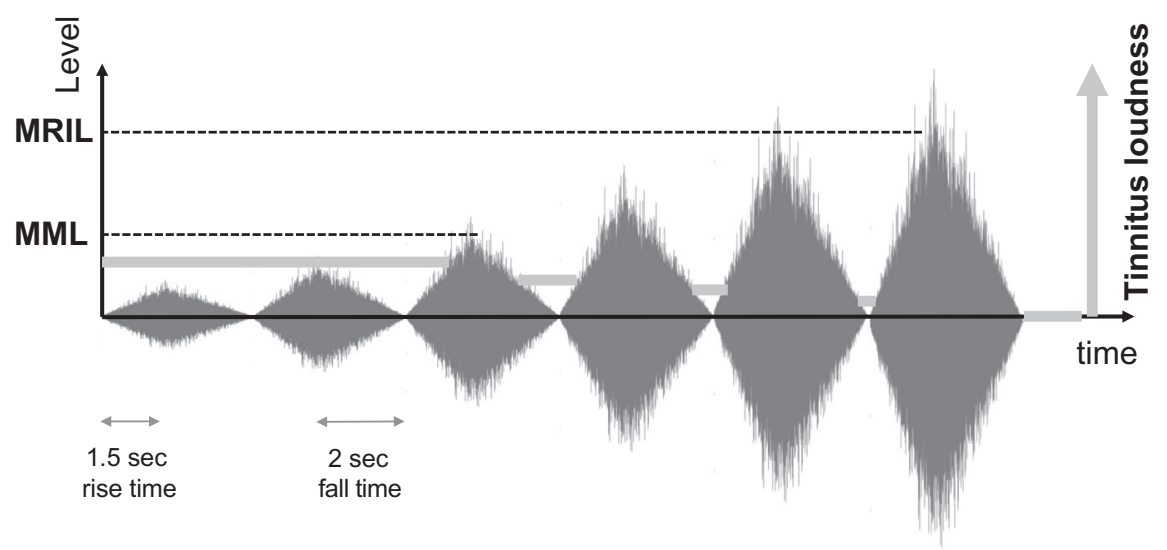

FIG. 2

Schematic representation of the pulsed stimulation used in the present study. The continuous stimulus has a fixed-rise time of $1.5 \mathrm{~s}$ and fall time of $2 \mathrm{~s}$. There is an apparent silent gap between each pulse. The MML is obtained by raising the intensity of the pulsed noise until the tinnitus is masked during the peak of the stimulation. The MRIL is obtained by further increasing the intensity until the tinnitus is just suppressed during the silent interval between two pulses. The red line represents the loudness of the tinnitus decreasing as the intensity of the stimulation increases. RI, residual inhibition; MML, minimum masking level; MRIL, minimum residual inhibition level.

$1.5 \mathrm{~s}$ and fall time of $2 \mathrm{~s}$ (Fig. 2) instead of a more abrupt rise and fall time used previously. This was done in order to deliver a more comfortable stimulation to the ears of the participants. Masker thresholds (MT), MMLs and MRILs were obtained during the same sequence presentation as follows: firstly, the ramped stimulus was presented binaurally at infra threshold levels and raised by $2 \mathrm{~dB}$ steps until the participants detected the narrowband noise (MT). Secondly, the level was raised by $3 \mathrm{~dB}$ steps until the tinnitus was masked during the stimulus presentation (MML) (Fig. 2). Thirdly, the level of the stimulus was further raised by 2 or $3 \mathrm{~dB}$ steps until the tinnitus was suppressed during the silent interval, between the fall time of the previous ramp and the rise time of the next ramp (MRIL). The maximum intensity of each stimulus did not exceed $96 \mathrm{~dB}$ SPL and all participants were asked to report at any time if the stimulus intensity caused discomfort. In the cases where this level was reached, the ongoing sequence was immediately stopped and we skipped directly to another frequency masker. Four different one octave width narrowband noises were used. The narrowband noise masker centered at $1 \mathrm{kHz}$ was used as a reference for all participants. The second frequency masker was around the middle of the hearing loss slope, the third was at the characteristic frequency of the tinnitus of each participant, and the fourth was white noise. Hearing loss slope is an analogy of a ski slope: in some cases of sensorineural hearing loss (c.f. presbycusis) the hearing deficit represented on the audiogram resembles a slope that is characterized by ascending thresholds as the frequency increases. If the participants did not exhibit any hearing loss 
slope, the $4 \mathrm{kHz}$ narrowband noise masker was arbitrarily used as the HL slope frequency. For a subgroup of the tinnitus participants $(n=10$, Table 1$)$, the MML and MRIL measurements were performed for both the tinnitus and an external sound mimicking tinnitus (e.g. similar frequency and intensity). For example, a participant with tinnitus at $4 \mathrm{kHz}$ and $7 \mathrm{~dB}$ SL as measured in the pitch-matching and loudnessmatching tasks was first tested for all the MML and MRIL conditions with the tinnitus as the target. Afterward, the participant was tested again but with an external sound set at the same loudness and pitch as his tinnitus. In his case, this would be a $4 \mathrm{kHz}$ pure tone presented continuously at $7 \mathrm{~dB}$ SL. This external pure tone was thus the new target for all the MML and MRIL conditions. This allowed a within group comparison of the MMLs and MRILs: MMLs and MRILs when the tinnitus was the target compared to that when an external sound mimicking tinnitus was the target. The external sound mimicking tinnitus was always a pure tone regardless of the tinnitus type that best matched the tinnitus in the matching task. For the control group, a continuous $4 \mathrm{kHz}$ pure tone set at $10 \mathrm{~dB}$ SL was presented continuously in order to mimic as closely as possible a real tinnitus sensation in terms of pitch (Schecklmann et al., 2012) and loudness (Fournier et al., 2019; Goodwin and Johnson, 1980; Hallam et al., 1985; Tyler and Conrad-Armes, 1984). The simulated tinnitus signal was presented without interruption through headphones during the entire session of the psychoacoustic measurements for the CG (e.g, the MT, the MMLs, the MRILs) and only during the session when the simulated tinnitus was tested for the tinnitus subgroup (MMLs and MRILs). For the control group, the MT, the MML and MRIL measurements were obtained with the same narrowband noises as the ones presented for the tinnitus group: narrowband noises with a center frequency fixed at $1 \mathrm{kHz}$ (reference), $4 \mathrm{kHz}$ (target frequency), $8 \mathrm{kHz}$ (reference) and white noise. As the control participants were included in the study only if they presented normal hearing, the frequency of the hearing loss slope was replaced by a reference frequency at $8 \mathrm{kHz}$. For brevity, the within conditions of the tinnitus group are abbreviated as RT-TG and ST-TG throughout the article which stands for Real Tinnitus-Tinnitus Group for the former, and Simulated Tinnitus-Tinnitus Group for the latter. As the control group was only presented with a simulated tinnitus, only one abbreviation is used: ST-CG which stands for Simulated Tinnitus-Control Group.

In addition, all of these measures were performed with the narrowband maskers presented diotically (same sound perceived in both ears) and dichotically (by reversing the signal delivered in each ear, simply by multiplying the left signal by -1 ). The objective was to compare diotic and dichotic MMLs and MRILs in order to assess which method would be the most efficient, that is, requiring the lowest level of stimulation. Each participant underwent every measure twice (diotic and dichotic) and all conditions were randomized.

\subsubsection{Visual analog scales}

Visual analog scales (VAS) of tinnitus intensity were used during the procedure for the tinnitus group. The scales were $100 \mathrm{~mm}$ horizontal lines with the left and right extremes labeled, "very faint" and "very loud," respectively. Participants were required to give a first estimation of the intensity of their tinnitus at the beginning 
of the testing session before any sound was presented. Participants were asked to use this VAS only when partial residual inhibition was obtained in a condition. This happened 22 times corresponding to $14 \%$ of all MRIL conditions $(n=160)$.

\subsection{Procedure}

The experimental testing first started with a brief assessment of the medical history of each participant, as well as a qualitative description of their tinnitus, for the tinnitus participants. The aim of the study and an exhaustive explanation of the procedure was shared with all participants. All participants gave oral and written consent for their participation. Tinnitus participants were then asked to fill out a questionnaire regarding their tinnitus, their possible medical treatments if applicable, the presence of any hearing impairments and their overall distress regarding tinnitus. Hearing thresholds were first measured for all participants, controls and tinnitus participants. For the tinnitus group, pitch matching and loudness matching were then performed. The tinnitus participants were asked to rate the loudness of their tinnitus on a visual analog scale (VAS) before any other sound was presented. For the control group, the participants were presented a continuous $4 \mathrm{kHz}$ pure tone at an audible level. The intensity was then gradually decreased by $1 \mathrm{~dB}$ steps until the participant reported the sound to be just inaudible. The experimenter increased the signal $10 \mathrm{~dB}$ louder and informed the participant that the simulated tinnitus was set and would be played continuously until the experiment was over. The MT, MML and MRIL measurements using either a narrowband noise centered at one of three frequencies $(1 \mathrm{kHz}, 4$ and $8 \mathrm{kHz}$ for the CG, $1 \mathrm{kHz}, \mathrm{HL}$ slope and tinnitus frequency for the TG) or white noise began in a random order and all four frequencies were assessed diotically and dichotically in one sitting for all participants in both groups. The MT, MMLs and MRILs were measured until all the eight different configurations were tested. For a subgroup of tinnitus participants $(n=10)$, the same frequency conditions were tested again only in diotic presentation but for the simulated tinnitus as the target, that is, with the presence of an external sound mimicking tinnitus. At the end of the procedure, the results of the study were shared with the participants and the tinnitus participants were given a copy of the most comfortable/efficient audio sequence, if desired. The average time of the whole procedure was about $1 \mathrm{~h}$ for the control group and $1 \mathrm{~h}$ and a half for the tinnitus group.

\subsection{Statistical analysis}

First, chi-square tests were run to determine if the proportion of achievable MMLs and MRILs was significantly different between the two groups (TG vs CG) and between the two target conditions (RT vs ST) for the tinnitus subgroup. For the MMLs, chi-square analysis included Total MML and No MML as a within-subject variable. For the MRILs, the Chi-square analysis included Total RI, Partial RI and No RI as a within-subject variable. Partial residual inhibition was defined by a reduction of at least two data points on the visual analog scale after any given condition 
where total suppression of the tinnitus was not reached. In addition, all MML and MRIL measures were converted from sound pressure levels (dB SPL) to sensation levels (dB SL) by subtracting the masker thresholds at each frequency masker for each participant. This was done in order to account for the hearing threshold differences at each of the tested frequencies and to reduce any bias that can emerge from hearing threshold differences between tinnitus participants and normal hearing controls. Before any parametric analysis was conducted, a thorough examination of the normality of the distribution was tested thanks to Shapiro-Wilk tests. For any given parametric analysis, the significance did not fall under $P=0.1$ except for the distributions of the control group at the reference frequency (control group distribution for MMLs at reference frequency between diotic and dichotic $P>0.005$ and $P>0.002$, respectively). In this case only, a non-parametric analysis was conducted in order to respect statistical and scientific standards. It is important to note that the number of participants differs across analysis because some participants did not display MMLs and MRILs for all conditions. A repeated measures analysis of variance was conducted to investigate different masker thresholds between the two study samples. A mixed analysis of variance (ANOVA) was used in order to assess group differences regarding the type of measure (MML and MRIL), the phase of the masker (diotic and dichotic) and the target center frequency tested (HL slope, tinnitus frequency and white noise). Another repeated measures analysis of variance was conducted in order to further analyze the tinnitus subgroup only. The within subject factors included the type of measure (MML and MRIL), the phase of the masker (diotic and dichotic), the center frequency of the masker (HL slope, tinnitus frequency and white noise) and the different target (the tinnitus and the mimicking sound). Finally, a series of paired $t$-tests were conducted in order to precisely observe the differences between diotic and dichotic maskers across every frequency tested for each sample group.

\section{Results}

\subsection{Individual examples}

Fig. 3 displays eight individual examples including six tinnitus participants (Tinnitus participant 01, 02, 03, 04, 05 and 06) and two controls (Control participant 01 and 02). The individual data of all participants, tinnitus and controls, are available in the Supplementary material in the online version at https://doi.org/10.1016/bs.pbr. 2020.08.010 section (Tinnitus: Supplementary Fig. 1 in the online version at https:// doi.org/10.1016/bs.pbr.2020.08.010, Controls: Supplementary Fig. 2 in the online version at https://doi.org/10.1016/bs.pbr.2020.08.010). The hearing thresholds for each ear, the MMLs and the MRILs are displayed for each example. The star represents the tinnitus frequency and loudness as measured psychoacoustically by a pitch and loudness matching task. The tinnitus participant 01 was not tested with the simulated tinnitus target condition. For the control participants, the star represents the 

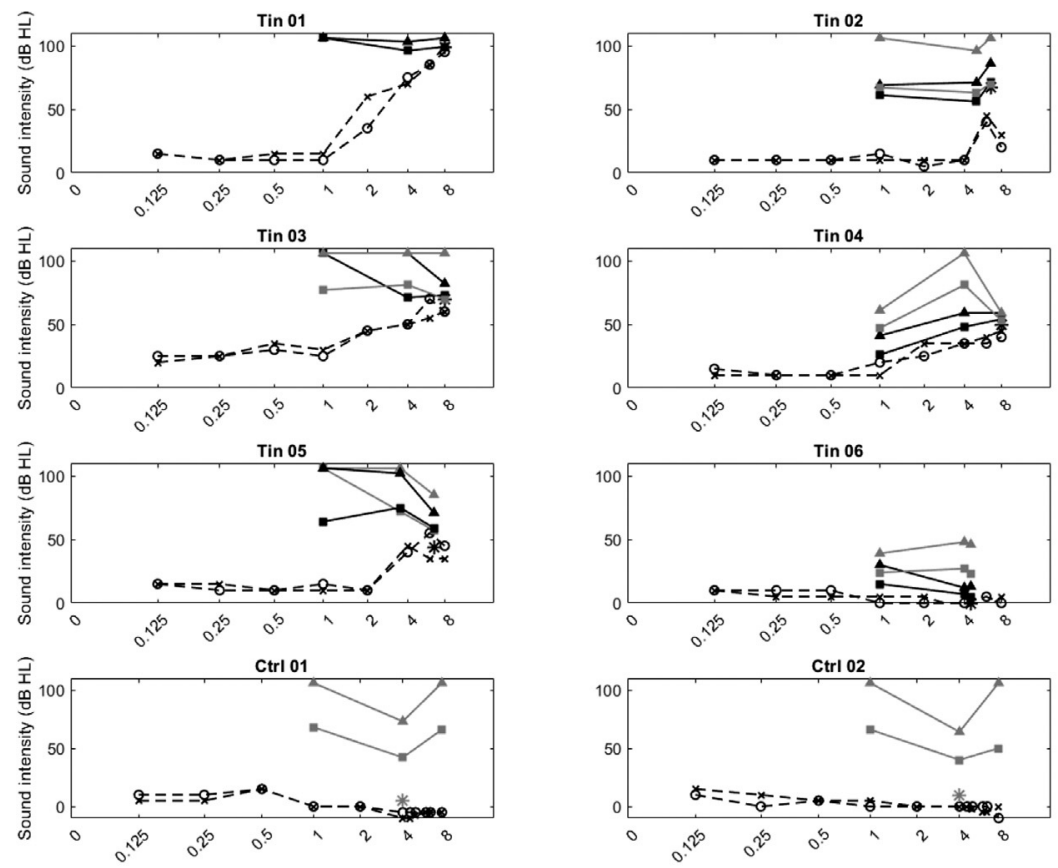

Frequency $(\mathrm{kHz})$

Frequency $(\mathrm{kHz})$

$-*-\cdot$ HT RE $-\bullet-\cdot$ HT LE $\quad * \quad \begin{aligned} & \text { Simulated tinnitus } \\ & \text { frequency and level }\end{aligned} \quad * \begin{aligned} & \text { Tinnitus frequency } \\ & \text { and level }\end{aligned} \longrightarrow$ MML ST $\longrightarrow$ MML RT-TG $\longleftarrow$ MRIL ST $\longrightarrow$ MRIL RT-TG

FIG. 3

The hearing thresholds, the MMLs and MRILs of eight participants: six tinnitus participants (with four from the tinnitus subgroups) and two control participants. HT, hearing thresholds; $\mathrm{RE}$, right ear; LE, left ear; MML RT-TG, minimum masking levels for the real tinnitus target for the tinnitus group only, MML ST, minimum masking levels for the simulated tinnitus target; MRIL RT-TG, minimum residual inhibition level for the real tinnitus target for the tinnitus group only; MRIL ST, minimum residual inhibition level for the simulated tinnitus target.

simulated tinnitus which was set by the experimenter at a frequency of $4 \mathrm{kHz}$ and at an intensity of $10 \mathrm{~dB}$ SL. The MMLs and MRILs that were tested but not achieved were attributed an arbitrary value of $106 \mathrm{~dB}$.

The Tinnitus participant 01 presented a bilateral hearing loss typical of presbycusis from 2 to $8 \mathrm{kHz}$ with a tinnitus frequency measured at $8 \mathrm{kHz}$ and $4 \mathrm{~dB} \mathrm{SL}$, which is expected for this type of hearing loss. The lowest MMLs and MRILs were obtained with the masker frequency centered at the tinnitus frequency, that is, $8 \mathrm{kHz}$ (4 and $13 \mathrm{~dB}$ SL, respectively). In his case, the MML and the MRIL were tested but not obtained with the masker centered at $1 \mathrm{kHz}$. The tinnitus participant 02 displayed a notch-type hearing loss centered at $6.5 \mathrm{kHz}$. Interestingly, the tinnitus in this participant was measured at the same frequency as the hearing notch loss, e.g. $6.5 \mathrm{kHz}$, and with an intensity of $27 \mathrm{~dB}$ SL. In his case, MMLs and MRILs were obtained for all three maskers with lower levels, in $\mathrm{dB}$ SL, for the maskers centered at the TF and 
the HL slope frequency. In addition, for this participant, the MML and MRIL were generally lower for the real tinnitus target compared to the simulated tinnitus target. The participants 03,04 and 05 all displayed mild bilateral hearing loss with a tinnitus frequency located in the hearing loss frequency region $(8 \mathrm{kHz}$ for participant 03 and $04,6.5 \mathrm{kHz}$ for participant 05 ). The intensity of the tinnitus was only a few decibels above the threshold (10, 10 and $9 \mathrm{~dB}$ SL, respectively) for all participants. In these participants, the tinnitus and the simulated tinnitus targets were both more easily masked and suppressed by the masker centered at the TF. In addition, it is clear for participants 04 and 05 that the real tinnitus was more easily masked and suppressed than the simulated tinnitus, that is, displayed lower levels of MML and MRIL. Interestingly, Tinnitus participant 06 had no measurable hearing loss at standard clinical frequencies $(0.125-8 \mathrm{kHz})$ for both ears. He also had a $4.55 \mathrm{kHz}$ tinnitus frequency measured at $5 \mathrm{~dB}$ SL. He is the tinnitus example with the most similarities with participants of the CG, that is, normal hearing thresholds at standard audiometric frequencies and a tinnitus very close to $4 \mathrm{kHz}$. In his case, the lowest MML value (10dB SL) was obtained for the frequency masker set at the tinnitus frequency and the lowest MRIL ( $\sim 18 \mathrm{~dB} \mathrm{SL})$ was obtained for both $4 \mathrm{kHz}$ and the TF $(4.5 \mathrm{kHz})$ maskers. For this tinnitus participant, the MMLs and MRILs were lower, in $\mathrm{dB}$ SL, when the real tinnitus was the target compared to when the simulated tinnitus was the target. All the controls behaved similarly during the task. As shown for Control participant $01 \& 02$, they had normal hearing thresholds at standard audiometric frequencies for both ears. The MML and MRIL values were lower when the masker frequency was centered at the simulated TF (e.g. $4 \mathrm{kHz}$ ). Most participants also displayed some level of MML at other frequencies $(1$ and $8 \mathrm{kHz})$ but their values were much higher than for the masker frequency centered at TF. These masking curves display a similar V-shape as expected with psychophysical tuning curves (Fournier et al., 2019; Kluk and Moore, 2006; Moore and Alcántara, 2001). In addition, the MRIL was obtained with the masker centered at TF: no MRILs were obtained with the $1 \mathrm{kHz}$ and $8 \mathrm{kHz}$ frequency maskers (this is why the values are displayed at $106 \mathrm{~dB}$ ). These results suggest that forward masking is even more bounded by frequency selectivity than masking when an external sound is the target.

\subsection{Distribution of MMLs and MRILs between groups}

Table 2 presents the frequency distribution of achievable MMLs and MRILs for the different stimulation conditions for both groups of participants. For the tinnitus group, MMLs were obtained for all participants when the narrowband noise masker was centered at the frequency of the hearing loss slope and white noise for both, diotic and dichotic conditions. For the masker centered at the TF, MMLs were obtained for all participants except one for the dichotic presentation only. The lowest proportion of MMLs obtained in the tinnitus group was for the masker centered at the reference frequency $(1 \mathrm{kHz})$ where 14 and 13 out of 20 participants obtained a measurable MML for the diotic and dichotic configurations, respectively. 
Table 2 Proportions of achievable MMLs and MRILs for each frequency masker conditions between the real tinnitus target for the tinnitus group $(n=20)$ and the simulated tinnitus target for the control group $(n=20)$.

\begin{tabular}{|c|c|c|c|c|c|c|c|c|c|c|}
\hline \multirow{2}{*}{$\begin{array}{l}\text { Type of } \\
\text { measure }\end{array}$} & \multirow{2}{*}{$\begin{array}{l}\text { Frequency of } \\
\text { masker }\end{array}$} & & \multicolumn{3}{|c|}{ Tinnitus group-real tinnitus } & \multirow[b]{2}{*}{ Frequency of masker } & \multicolumn{3}{|c|}{ Control group-simulated tinnitus } & \multirow[b]{2}{*}{ Chi-square } \\
\hline & & & $n$ & $n$ & $n$ & & $n$ & $n$ & $n$ & \\
\hline MML & $\begin{array}{l}\text { Reference }(1 \mathrm{kHz}) \\
\text { HL slope } \\
\text { Tinnitus frequency } \\
\text { White noise }\end{array}$ & $\begin{array}{l}\text { Diotic } \\
\text { Dichotic } \\
\text { Diotic } \\
\text { Dichotic } \\
\text { Diotic } \\
\text { Dichotic } \\
\text { Diotic } \\
\text { Dichotic }\end{array}$ & $\begin{array}{l}\text { Total MML } \\
14 \\
13 \\
20 \\
20 \\
\\
20 \\
19 \\
20 \\
20 \\
146(91 \%) \\
\end{array}$ & $\begin{array}{l}\text { No MML } \\
6 \\
7 \\
0 \\
0 \\
\\
0 \\
1 \\
0 \\
0 \\
14 \text { (9\%) } \\
\end{array}$ & & $\begin{array}{l}\text { Reference }(1 \mathrm{kHz}) \\
\text { Reference }(8 \mathrm{kHz}) \\
\begin{array}{l}\text { Target frequency } \\
(4 \mathrm{kHz})\end{array} \\
\text { White noise }\end{array}$ & $\begin{array}{l}\text { Total MML } \\
18 \\
18 \\
15 \\
16 \\
\\
20 \\
20 \\
20 \\
20 \\
147 \text { (92\%) }\end{array}$ & $\begin{array}{l}\text { No MML } \\
2 \\
2 \\
5 \\
4 \\
\\
0 \\
0 \\
0 \\
0 \\
13 \text { (8\%) } \\
\end{array}$ & & $\begin{array}{l}P \text {-value } \\
\text { n.s. } \\
\text { n.s. } \\
=0.02 \\
=0.04 \\
\text { n.s. } \\
\text { n.s. } \\
\text { n.s. } \\
\text { n.s. } \\
\text { n.s. }\end{array}$ \\
\hline MRIL & $\begin{array}{l}\text { Reference (1 kHz) } \\
\text { HL slope } \\
\text { Tinnitus frequency } \\
\text { White noise }\end{array}$ & $\begin{array}{l}\text { Diotic } \\
\text { Dichotic } \\
\text { Diotic } \\
\text { Dichotic } \\
\text { Diotic } \\
\text { Dichotic } \\
\text { Diotic } \\
\text { Dichotic }\end{array}$ & $\begin{array}{l}\text { Total MRIL } \\
8 \\
6 \\
12 \\
13 \\
\\
11 \\
14 \\
17 \\
15 \\
96 \text { (60\%) }\end{array}$ & $\begin{array}{l}\text { Partial MRIL } \\
1 \\
3 \\
5 \\
4 \\
\\
4 \\
3 \\
2 \\
1 \\
23(14 \%)\end{array}$ & $\begin{array}{l}\text { No MRIL } \\
11 \\
11 \\
3 \\
3 \\
\\
5 \\
3 \\
1 \\
4 \\
41(26 \%)\end{array}$ & $\begin{array}{l}\text { Reference }(1 \mathrm{kHz}) \\
\text { Reference }(8 \mathrm{kHz}) \\
\text { Target frequency } \\
(4 \mathrm{kHz})\end{array}$ & $\begin{array}{l}\text { Total MRIL } \\
1 \\
1 \\
1 \\
3 \\
\\
18 \\
18 \\
13 \\
13 \\
68 \text { (42.5\%) }\end{array}$ & $\begin{array}{l}\text { Partial MRIL } \\
0 \\
0 \\
0 \\
0\end{array}$ & $\begin{array}{l}\text { No MRIL } \\
19 \\
19 \\
19 \\
17 \\
\\
2 \\
2 \\
7 \\
7 \\
92(57.5 \%)\end{array}$ & $\begin{array}{l}P \text {-value } \\
\\
<0.02 \\
<0.02 \\
\\
<0.001 \\
<0.001\end{array}$ \\
\hline
\end{tabular}

Significant P-values are in bold. 
For the control group, all the participants $(n=20)$ obtained MMLs when the masker was centered at the target frequency $(4 \mathrm{kHz})$ and when using white noise for both diotic and dichotic conditions. A large proportion of controls (18 out of 20) obtained MMLs when the masker was centered at $1 \mathrm{kHz}$ again for both phase configurations. The lowest proportion of MMLs for the control group was obtained with the masker centered at $8 \mathrm{kHz}$ with only 15 participants achieving MML (75\%) diotically and 16 participants $(80 \%)$ dichotically. Overall, MMLs were obtained in $90 \%$ of all conditions for the tinnitus group and $91.8 \%$ for the control group, thus yielding a similar proportion of MMLs between the two groups. The tinnitus group displayed a significantly higher proportion of MML only for the HL slope frequency condition for both diotic and dichotic presentations, $X^{2}(1,40)=5.7, P=0.017$ value and $X^{2}(1,40)=4.4, P=0.035$, respectively.

For the MRILs, none of the stimulation conditions provided residual inhibition for all the participants in any of the two groups. For the TG, the white noise condition provided residual inhibition (partial and total RI merged) for 19 participants (95\%) in the diotic presentation and 16 in the dichotic presentation. Seventeen participants $(85 \%)$ obtained an MRIL when the NBN was centered at the HL slope frequency in both diotic and dichotic conditions as well as at the tinnitus frequency in the dichotic configuration. Only 9 out of 20 participants (45\%) obtained MRILs when the center frequency of the masker was set at the reference frequency $(1 \mathrm{kHz})$, regardless of the phase configuration of the masker.

For the control group, the highest proportion of RI $(n=18,90 \%)$ was obtained with the NBN masker centered at the target frequency $(4 \mathrm{kHz})$ in both phase configurations. The lowest proportion of control participants displayed residual inhibition when the NBN was centered at the $1 \mathrm{kHz}$ and $8 \mathrm{kHz}$ reference frequencies with only $5 \%$ and $15 \%$ of participants displaying some level of inhibition at those frequencies, respectively. At last, 13 participants of the control group $(65 \%)$ exhibited some extent of RI when the white noise stimulation was used, regardless of the phase configuration of the masker.

Overall, MRILs (partial and total RI merged) were obtained in $74.3 \%$ of all conditions for the tinnitus group but only $42.5 \%$ for the control group, suggesting a strong discrepancy between the poststimulation suppression of tinnitus in the TG and the poststimulation suppression of an external sound with similar characteristics (pitch and loudness) in the CG. Indeed, the total proportion of MRIL was significantly higher for the TG than for the CG, $X^{2}(1320)=47.3, P<0.001$. The proportion of MRIL was significantly higher for the TG for almost all conditions with the exception of the masker centered at the TF and the white noise for the dichotic presentation only (Table 2).

\subsection{Between subject's effects}

\subsubsection{Threshold differences between TG and CG}

A repeated measures analysis of variance was conducted on the noise masker thresholds with the group as the between-subjects factor (TG, CG), and the center frequency of the masker as the within-subjects factor (Reference, HL slope, tinnitus 

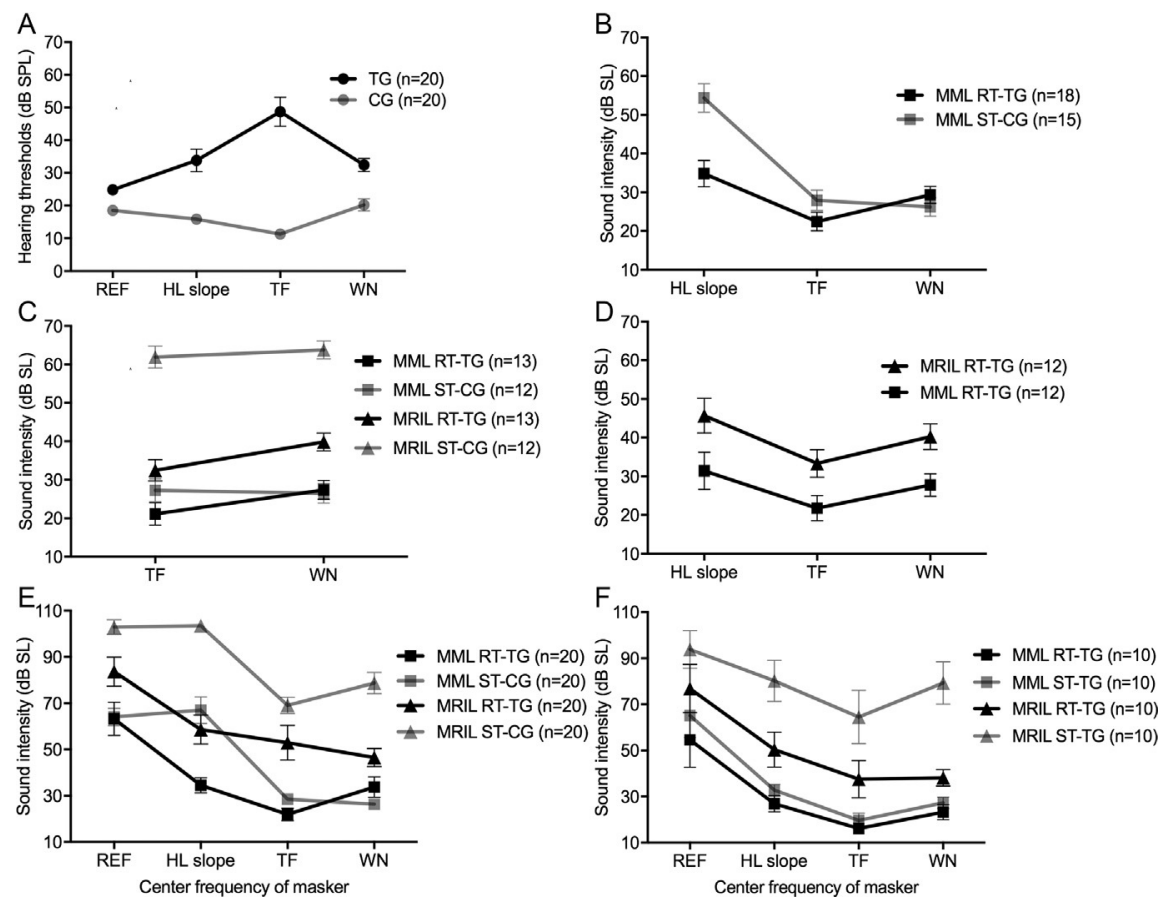

FIG. 4

(A) Hearing thresholds for the different maskers for the tinnitus group (TG) and the control group (CG). (B) MMLs between the two targets (RT, real tinnitus; ST, simulated tinnitus) of the two different groups (CG and TG) for three masker conditions. Only participants with measurable MML at all three masker frequencies were included here. (C) MMLs and MRILS between the TG with the RT target and the CG with the ST target for the two conditions with the most measurable MMLs and MRILs across both groups. (D) Comparison of MML and MRIL for the TG when the RT is the target for three masker conditions. (E) MMLs and MRILs between the TG with the RT target and the CG with the ST target for all conditions including the extreme values (No measurable MML and MRIL) for both groups. (F) MMLs and MRILs between the two targets (ST vs RT) for the tinnitus subgroup for all masker conditions including the extreme values (No measurable MML and MRIL). The error bars represent the Standard Error of the Mean (SEM).

frequency and white noise). As expected, a significant interaction between Groups and Frequency was found, $[F(3,114)=39.6, P<0.001$, $]$ which demonstrates the differences in terms of thresholds between the normal hearing controls (CG) and the tinnitus participants (TG). An illustration is available (Fig. 4A). The hearing threshold mean values are available in Supplementary material in the online version at https://doi.org/10.1016/bs.pbr.2020.08.010. A series of post hoc tests were conducted in order to further investigate the interaction observed between the two groups for each frequency tested. Despite the interaction observed above, 
independent sample $t$-tests with Bonferroni correction for multiple comparisons showed significant differences for every frequency tested (all $P s<0.01$ ).

\subsubsection{MML differences between TG and CG}

The analysis included the groups as the between-subjects factor (TG, CG), and two within-subjects factors composed of the center frequency of the masker (HL slope, tinnitus frequency, white noise) and the phase configuration of the masker (diotic, dichotic). The $1 \mathrm{kHz}$ reference frequency masker condition was not included in this analysis because it had a low proportion rate in the tinnitus group (Table 2). A significant interaction between the Groups and Frequency was found $[F(2,62)=$ 15.05, $P<0.001$ ] (Fig. 4B). The MML mean values are available in Supplementary material in the online version at https://doi.org/10.1016/bs.pbr.2020.08.010. These results comfort previous findings (Fournier et al., 2018) demonstrating minimal MMLs when the masker frequency is centered at the tinnitus frequency and/or frequencies of the hearing loss region for the TG. However, no significant effect of the phase of the masker (diotic vs dichotic) was found $[F(1,31)=0.084, P=0.77]$. The absence of the phase configuration effect was somewhat expected as we removed the $1 \mathrm{kHz}$ frequency masker condition from the analysis. No other significant interaction was found.

\subsubsection{MRIL differences between TG and CG}

Since the proportion of MRILs in some frequencies was very low in the CG, we analyzed the group differences of MMLs and MRILs only for the two masker frequencies that encompassed a maximum proportion of participants for both groups (namely, TF and WN). A repeated measures ANOVA that included the phase configuration of the masker (diotic, dichotic), the type of measure (MML, MRIL) and the center frequency of the masker (TF, WN) as the within-subjects factors, and the groups as the between-subjects factor (TG, CG) was conducted. A significant interaction between the Groups and the Type of measure was found $[F(1,23)=90.95$, $P<0.001]$, which demonstrates group differences in MMLs and MRILs between TG and CG (Fig. 4C). The MML and MRIL mean values are available in Supplementary material in the online version at https://doi.org/10.1016/bs.pbr.2020.08. 010. Post hoc tests revealed a significant mean difference of $-6.6 \mathrm{~dB}$ (SD: 3.2) for MML values between TG and CG when the masker was set to target the tinnitus frequency $[T(37)=-2.01, P=0.04]$ and a non-significant mean difference of $3.4 \mathrm{~dB}$ (SD: 2.9) when the masker was white noise $[T(37)=1.16, P=0.25]$. For the MRILs, a significant mean difference of $-31 \mathrm{~dB}$ (SD: 3.9) and $-22 \mathrm{~dB}$ (SD: 3.2) between the two groups was found when the center frequency was at the $\mathrm{TF}[T(31)=-7.84$, $P<0.001]$, and with white noise $[T(27)=-6.86, P<0.001]$, respectively. MRILs were much higher for the control group when the frequency of the masker was centered at the tinnitus frequency (65.1 dB SL for the CG vs $34.4 \mathrm{~dB}$ SL for the TG), as well as when the masker was white noise $(63.5 \mathrm{~dB}$ SL for the CG vs $41.6 \mathrm{~dB}$ SL for 
the TG). Overall, these results show how MMLs and MRILs are obtained at higher values when the target is an external sound mimicking tinnitus in the control group compared to when a real tinnitus is the target in the tinnitus group. Interestingly, these differences are significantly larger for the MRILs than for the MMLs.

In addition, an ANOVA that included the groups as the between-subjects factor (TG, CG) and the type of measure (MML, MRIL) and the frequency of the masker (Reference, HL slope, tinnitus frequency, white noise) as the within-subjects factors, was run for the diotic presentation only. All values were included even when MML and MRIL were not achievable: in these cases, the value was set at $106 \mathrm{~dB}$ SL. A significant main effect of the type of measure was found $[F(1,38)=289.2$, $P<0.001]$ as well as a significant main effect of the frequency of the masker $[F(3,114)=61.9, P<0.001]$. The difference between the two groups was also significant, $[F(1,38)=18.4, P<0.001]$. Importantly, there was a significant triple interaction between the group, the measure and the frequency $[F(3,114)=4.2$, $P=0.008$ ] (Fig. 4E). The MML and MRIL mean values are available in Supplementary material in the online version at https://doi.org/10.1016/bs.pbr.2020.08. 010. Post hoc tests corrected for multiple comparisons revealed only one significant MML difference between the two groups for the HL slope frequency condition $[T(38)=-4.9, P<0.001]$. For the MRIL, post hoc tests corrected for multiple comparisons revealed two significant differences between the two groups, one for the white noise masker, $[T(38)=6.1, P<0.001]$ and the other for the HL slope frequency masker, $[T(38)=6.8, P<0.001]$. For the $1 \mathrm{kHz}$ reference masker, the MRIL difference between the two groups was marginally significant after multiple comparisons correction, $[t(38)=-2.7, P=0.072]$.

\subsection{Within subject's effects}

\subsubsection{Distribution of MMLs and MRILs within a tinnitus subgroup}

As mentioned earlier, a subgroup of 10 tinnitus participants (Table 3) was tested with the MML and MRIL task but with a simulated tinnitus as the target (abbreviated as ST, an external sound at the tinnitus frequency and intensity), in addition to the MML and MRIL task when their real tinnitus (RT) was the target (Fig. 4F). The MML and MRIL mean values are available in Supplementary material in the online version at https://doi.org/10.1016/bs.pbr.2020.08.010. We then compared the proportion of total, partial or no MML and MRIL between the two target conditions (e.g. RT vs ST), for each of the frequency masker conditions. For the MMLs, the distribution was similar for both target conditions with all participants obtaining MML values for the HL slope frequency masker, the tinnitus frequency masker and the white noise masker. Seven participants out of 10 obtained MMLs for the $1 \mathrm{kHz}$ frequency masker, again, for both target conditions. Overall, MMLs were obtained in $92.5 \%$ of all conditions for both targets (Table 3). The proportion of achievable MMLs between the two targets was not significantly different for any of the frequency masker conditions (all $P s>0.05$ ). 
Table 3 Proportions of achievable MMLs and MRILs for each frequency masker conditions between the real tinnitus and the simulated tinnitus as the target for the tinnitus subgroup $(n=10)$.

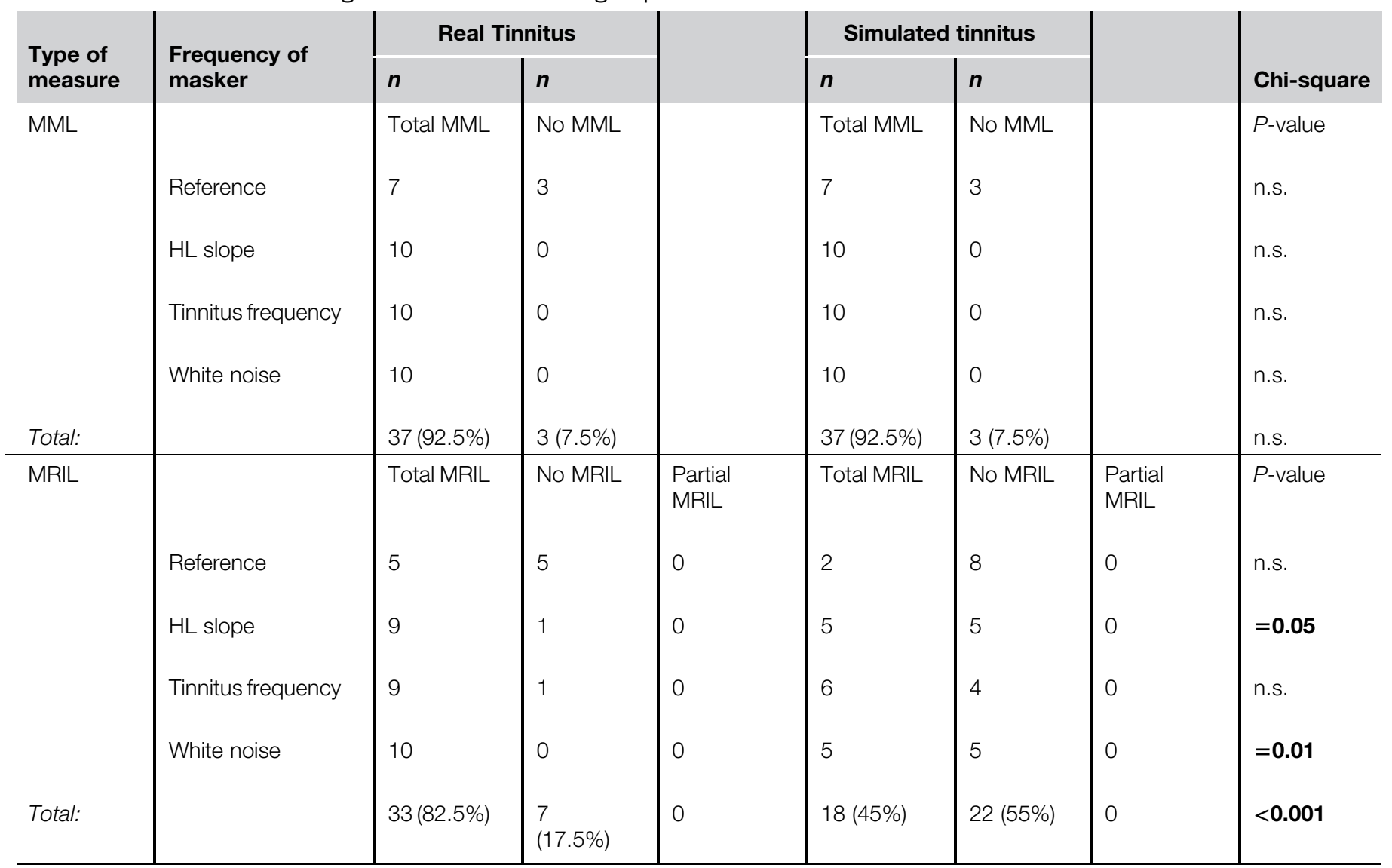


For the MRILs, the two target conditions displayed very different distributions across frequency masker conditions. Indeed, for the real tinnitus (RT) conditions, MRILs were obtained for all participants when using the white noise masker and for nine out of ten participants when using the HL slope frequency masker and the TF masker. The lowest proportion of MRILs for this target condition was obtained using the reference frequency masker with only 5 out of 10 MRILs obtained in these participants. For the simulated tinnitus (ST) target conditions, the proportion of MRILs across frequency conditions was quite low with only 6 , 5, 5 and 2 participants achieving MRIL out of 10 participants for the TF, white noise, HL slope and reference $1 \mathrm{kHz}$ masker, respectively. Overall, an MRIL was found in $82.5 \%$ of all frequency masker conditions when the real tinnitus was the target compared to $45 \%$ when the target was the simulated tinnitus, suggesting again a strong discrepancy between the poststimulation suppression of tinnitus and the poststimulation suppression of an external sound with similar characteristics (pitch and loudness). The proportion of achievable MRILs was significantly higher for the tinnitus target for the white noise masker and only marginally significant for the HL slope frequency, $X^{2}(1,20)=6.7, P<0.01$ and $X^{2}(1,20)=3.8, P=0.05$, respectively. For all the frequency masker conditions merged, the proportion of achievable MRILs for the tinnitus target was significantly higher than for the simulated tinnitus, $X^{2}(1,80)=12.2, P<0.001$.

\subsubsection{Effect of the target to mask (RT and ST) on MMLS and MRILS of TG}

In order to assess possible differences in MMLs and MRILs between the two different targets, paired-sample $t$-tests were run for all conditions merged, that is the conditions where MMLs and/or MRILs were obtained for both the real tinnitus (RT) and the simulated tinnitus (ST) target conditions, $(n=35$ and $n=18$ conditions, respectively) (Fig. $5 \mathrm{~A}$ ). For the 35 conditions with measurable MMLs for both targets, a significant mean MML difference of $5.8 \mathrm{~dB}$ (SD: 9.7) was found between the two, $[T(34)=3.55, P=0.001]$. This result suggests that tinnitus is more easily maskable (e.g. requires a lower level of masking) than an external sound with similar characteristics, that is, similar pitch and loudness. For the 18 conditions with measurable MRILs for both targets, a significant mean MRIL difference of $14.8 \mathrm{~dB}$ (SD: 15.2) was found between the two, $[T(17)=2.64$, $P<0.001$ ] (Fig. 5A). This result suggests that tinnitus is more easily suppressed, that is, MRIL requires a lower level of stimulation than the level required to produce a similar phenomenon for an external sound with similar characteristics. Overall, these results suggest that masking and RI are more difficult to obtain (e.g. require higher levels of stimulation) when the target is an external sound in comparison to a real tinnitus in a single patient. Finally, for the 18 conditions with measurable MRILs for both target conditions, there were good correlations between the MMLs and the MRILs (Fig. 5B) for both targets (RT and ST). 


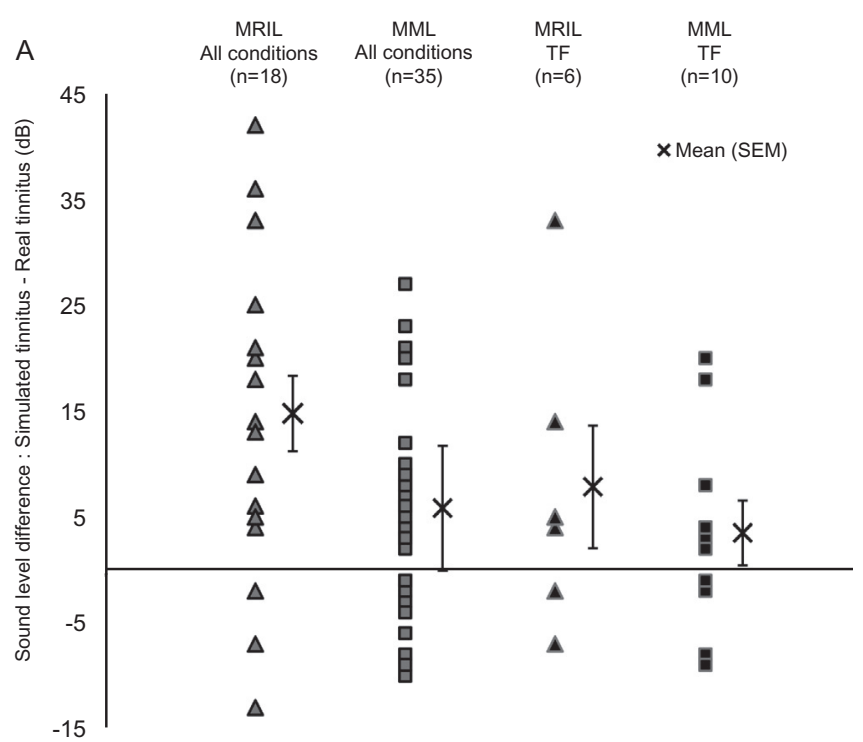

B

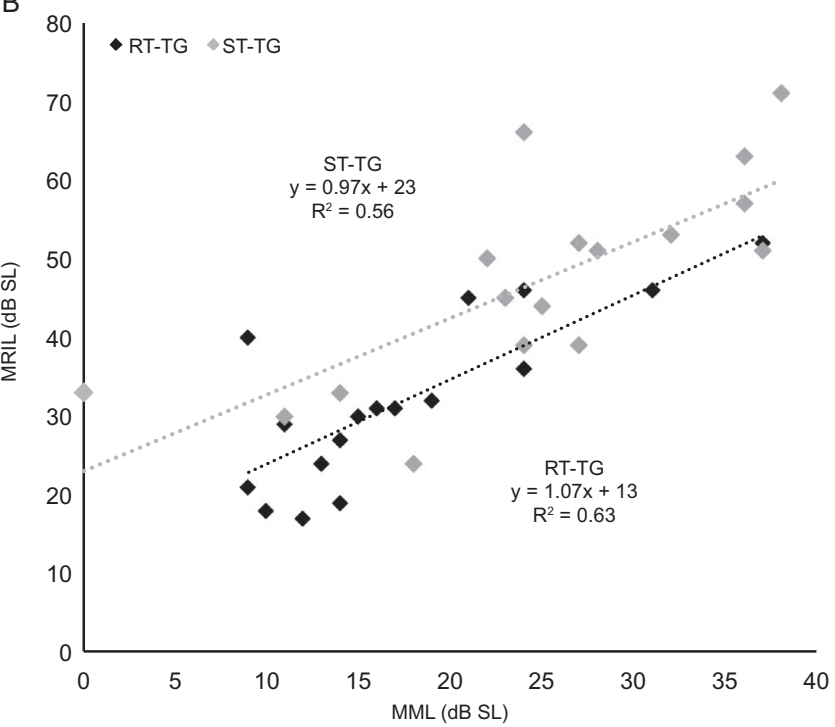

FIG. 5

(A) Sound level difference in MML and MRIL between the two targets (real tinnitus vs simulated tinnitus) for the tinnitus subgroup when all maskers conditions are merged and for the tinnitus frequency masker only. Only conditions where MMLs were obtained in the two target configurations were included here. This is also true for the MRILs. (B) Correlations between the MMLs and the MRILs for both targets for the tinnitus subgroup. Only measurable MRILs for both target conditions were considered here. The error bars represent the standard error of the mean (SEM). 


\subsubsection{Effect of frequency on MMLs and MRILs of TG and of TG subgroup}

A repeated measures ANOVA that included the phase of the masker (diotic, dichotic), the type of measure (MML, MRIL) and the frequency of the masker (HL slope, tinnitus frequency, white noise) was conducted for when the tinnitus was the target only $(n=12)$. This analysis was run only on participants with measurable MMLs and MRILs at those masker frequencies. A significant main effect of the type of measure was found $[F(1,11)=73.51, P<0.001]$ as well as a significant main effect of the frequency of the masker $[F(2,22)=5.51, P<0.01]$. Post hoc tests revealed significant mean differences between each frequency and each type of measure. MMLs and MRILs were minimal when the noise was centered at the tinnitus frequency in comparison to the other frequencies tested (all $P s<0.001$ ) which confirms previous work (Fournier et al., 2018) (Fig. 4D). The MML and MRIL mean values are available in Supplementary material in the online version at https://doi.org/10.1016/bs.pbr.2020.08.010. A masker centered at the tinnitus frequency showed significantly $(P<0.001)$ lower MMLs than other frequency conditions. This was also true for the MRILs $(P<0.001)$. The white noise configurations showed significantly lower MMLs and MRILs $(P<0.001)$ compared to a masker centered at the hearing loss slope frequency. These results suggest that the closer the frequency of the masker is to the tinnitus frequency, the lower the intensity of the stimulation is required to successfully mask and suppress the tinnitus. However, there was no significant effect of the different phase configurations of the masker $[F(1,11)=0.25, P=0.63]$.

In addition, a repeated measures ANOVA that included the type of measure (MML, MRIL), the type of target (RT, ST) and the frequency of the masker (Reference, HL slope, tinnitus frequency, white noise) for the diotic presentation only was run on the tinnitus subgroup. All values were included even when MML and MRIL was not achievable: in these cases, the value was set at $106 \mathrm{~dB}$ SL. A significant main effect of the type of measure was found $[F(1,9)=67.1$, $P<0.001]$ as well as a significant main effect of the frequency of the masker $[F(3,27)=18.5, P<0.001]$ and a main effect of the target $[F(1,9)=49.1, P<0.001]$. In addition, a significant interaction between the target and the measure was found $[F(1,9)=23.4, P=0.001]$ (Fig. 4F). Post hoc tests corrected for multiple comparisons revealed only significant mean MRIL differences for the white noise $[t(9)=-5.3$, $P<0.001]$ and for the HL slope frequency $[t(9)=-3.7, P=0.005]$ conditions. There were no significant differences for the MMLs.

\subsection{Diotic vs dichotic phase configurations}

As seen above, the phase configurations of the masker were always kept as a within subject factor in every statistical analysis. Still, none of the analysis revealed a significant main effect or interaction involving the different phase configurations. Fig. 6A displays the individual achievable MMLs between the diotic and dichotic conditions for all conditions merged for both groups, that is when the target is the tinnitus for the tinnitus group (RT-TG, $n=71$ ) and when the target is the simulated 

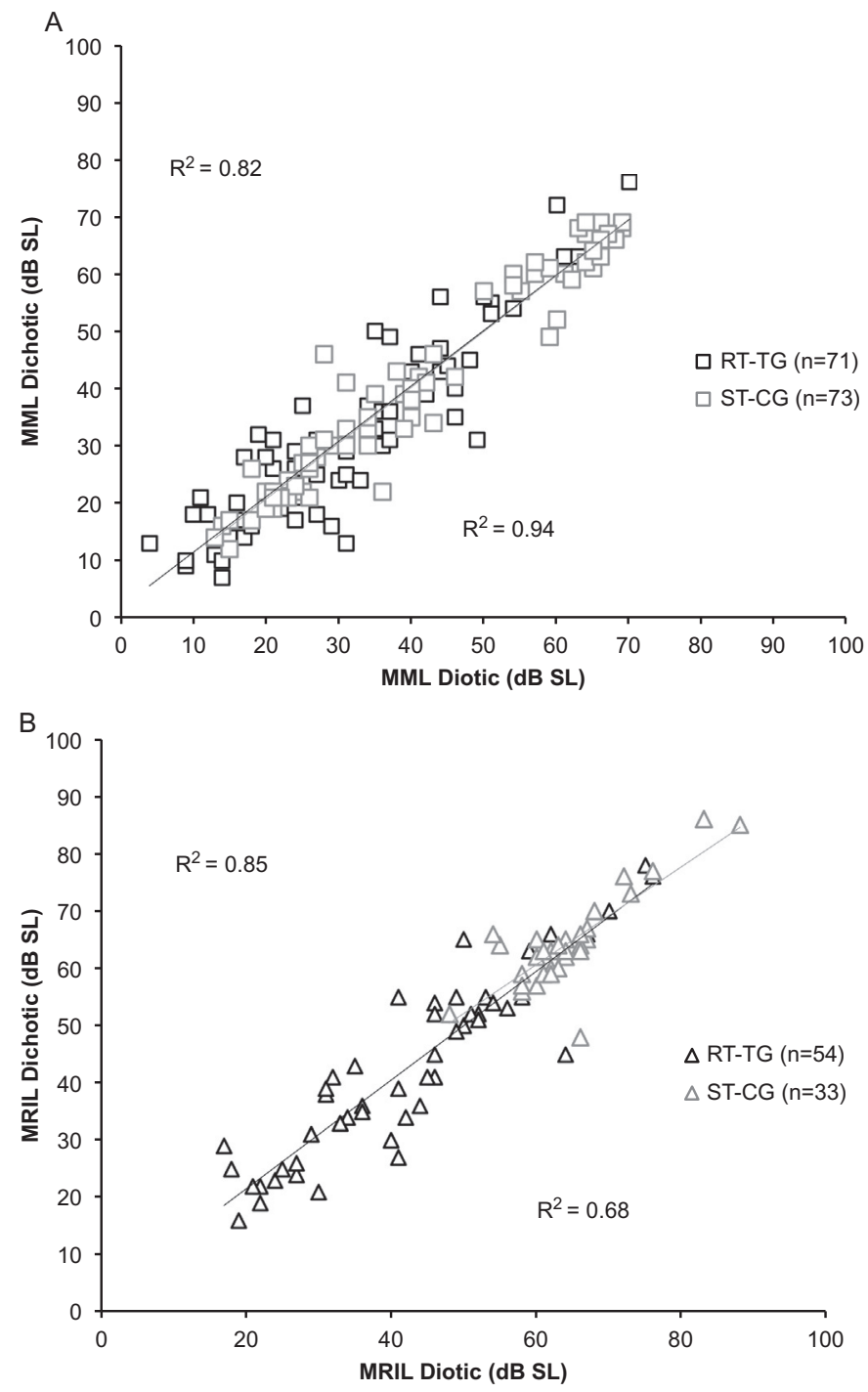

FIG. 6

$\overline{\text { Individual achievable MMLs (A) and MRILs (B) between the diotic and dichotic conditions for }}$ all conditions merged for both groups, that is when the target is the tinnitus for the tinnitus group and when the target is the simulated tinnitus for the control group.

tinnitus for the control group (ST-CG, $n=73$ ). The correlations between the diotic and dichotic phase configurations were really high for both, the RT-TG $\left(\mathrm{R}^{2}=0.82\right)$ and the ST-CG $\left(\mathrm{R}^{2}=0.94\right)$. Fig. $6 \mathrm{~B}$ displays the individual achievable MRILs for all conditions merged between the diotic and dichotic conditions for both groups, 
that is when the target is the tinnitus for the tinnitus group (RT-TG, $n=54$ ) and when the target is the simulated tinnitus for the control group (ST-CG, $n=33$ ). The correlation between the diotic and dichotic phase configurations was high for the RT-TG $\left(\mathrm{R}^{2}=0.85\right)$ and fair for the ST-CG $\left(\mathrm{R}^{2}=0.68\right)$. The mean MML difference between the diotic and dichotic phase configurations for all the merged conditions was of $0.7 \mathrm{~dB}$ (SD: 6.8) and 0.2 $\mathrm{dB}$ (SD: 4.5) for the RT-TG and the ST-CG, respectively. The mean MRIL difference between the diotic and dichotic phase configurations for all the merged conditions was of $0.4 \mathrm{~dB}$ (SD: 6.2 ) and $0.1 \mathrm{~dB}$ (SD: 4.8) for the RT-TG and the ST-CG, respectively (paired sample $t$-test, all $P s>0.3$ ). Overall, no significant differences were found between the diotic and dichotic conditions for either the RT-TG or the ST-CG. The good correlations between the two-phase configuration conditions suggest a good within session reliability for the MML and the MRIL measurements.

\section{Discussion}

The main objective of the present study was to explore the differences between the poststimulus suppression of tinnitus and of an external sound with similar characteristics (frequency and loudness). These phenomena refer to residual inhibition for the former, and forward masking for the latter. To our knowledge, differentiating the two phenomena experimentally in humans has never been attempted before. This was done by using a new technique developed at our laboratory to measure residual inhibition, that is, the minimum residual inhibition level (Fournier et al., 2018). This technique used pulsed narrowband noise stimulation with fixed stimulation duration $(\sim 3 s)$ and gap duration $(\sim 1 s)$ that allows to measure the level of stimulation required to produce suppression of tinnitus during the gap of silence. The MRIL can be measured with different frequency maskers quickly and can give indication about the best frequency to stimulate in order to produce residual inhibition, that is, the frequency masker that will produce the lowest MRIL in dB SL. For the tinnitus participants, we thus used this technique when the tinnitus was the target to measure the minimum level of a masker required to produce $1 \mathrm{~s}$ of residual inhibition (MRIL). In addition, we also used the same technique but when an external sound mimicking tinnitus characteristics (frequency and loudness) was the target in both, a subgroup of tinnitus participants and a control group in order to measure the level required to produce forward masking suppression of a similar duration. This way, we are able to first compare if the suppression of tinnitus and of the external sound is similarly achievable and if so, if they are equally distributed between frequency masker conditions. Finally, we could assess if they are achieved at a similar or different level of stimulation across frequency masker conditions.

Overall, the results showed that MRIL was more achievable for tinnitus than for an external sound mimicking tinnitus for both within group comparisons (tinnitus vs simulated tinnitus for the TG) and between group comparisons, that is, tinnitus as the target for the tinnitus group vs the simulated tinnitus for the control group. 
Some extent of RI was achievable in $74 \%$ (14\% partial RI and $60 \%$ complete RI) of the cases when tinnitus was the target for the tinnitus group compared to $45 \%$ and $43 \%$ (complete RI only, no partial RI) of the cases when the target was an external sound for the tinnitus subgroup and the control group, respectively. In addition, for the control group, MRIL was achievable almost exclusively for the frequency masker centered at the target frequency and the white noise. Conversely, when tinnitus was the target, MRIL was achievable for at least $85 \%$ of all tinnitus participants for most frequency maskers with the exception of the $1 \mathrm{kHz}$ reference masker, which displays a lower proportion close to $45 \%$ of achievable MRILs. This result is consistent with previous studies showing that residual inhibition is more efficient when the masker is at or close to the tinnitus frequency region (Fournier et al., 2018; Roberts et al., 2006, 2008). These results also suggest that, similar to tinnitus masking, the residual inhibition of tinnitus has a lower frequency selectivity compared to the poststimulus suppression of an external sound. Moreover, the pulse noise level required to reach residual inhibition was significantly lower for the tinnitus target than for the external sound target, for both the TG and the CG. Indeed, the mean level difference was close to $30 \mathrm{~dB}$ between the tinnitus target of the TG and the simulated tinnitus of the $\mathrm{CG}$, and close to $15 \mathrm{~dB}$ between the two targets for the tinnitus subgroup (the lower difference in TG likely results from hearing loss and cochlear recruitment). In summary, these results suggest a strong discrepancy between the poststimulation suppression of tinnitus (RI) and the poststimulation suppression of an external sound (forward masking). These results and their implications will be discussed in turn.

\subsection{Implications for the mechanisms of tinnitus \\ 4.1.1 Frequency selectivity of the suppression}

The phenomenon of tinnitus masking (TM) and residual inhibition (RI) of tinnitus are two ways to investigate how external sounds interact with tinnitus: TM provides insight on the fusion between external sound activity and tinnitus related activity while RI provides insight on how the external sound might suppress the tinnitus related activity for a period of time. Masking of an external sound can be summarized as the interaction or the fusion of the activity produced by the masker and the signal. Indeed, in this case, the interaction in play is highly constrained by the "place" where each component resides at the cochlear level in a way that when the frequency of the masker and the signal are far apart, the masking becomes no longer possible (or at very high levels of stimulation). In this case, the fusion of the masker and the signal is not achieved. This feature is also true for forward masking with the specificity that the interaction is made more durable in the form of a postactivation suppression of the neural activity. Indeed, forward masking has also been shown to be bounded by frequency selectivity (Oxenham and Plack, 2000). Tinnitus does not abide by these rules, as a $1 \mathrm{kHz}$ external masker is able to mask and in some cases even suppress an $8 \mathrm{kHz}$ tinnitus during a certain period of time. As seen in our results, close to none of 
our controls (5\%) obtained MRIL when the target to suppress was not encompassed in the bandwidth of the masker. However, a fair proportion of the tinnitus participants exhibited a form of RI when the masker was centered outside the region of their tinnitus with $45 \%$ when it was centered at the reference frequency $(1 \mathrm{kHz})$ and $85 \%$ when centered at the hearing loss slope frequency. The fusion between the tinnitus-related activity and the activity generated by the masker is not located at the cochlear level, nor at the level of the acoustic nerve, as it is only a "neural copy" of the activity generated in the cochlea. Thus, the interaction must reside at a higher level in the auditory centers. One might ask what happens in the auditory centers? From what we can say for sure: the activity related to the presentation of the masker is sculpted by the cochlea and is rendered quite shallow (the spreading of the tonotopic activity depends on the intensity). However, the tinnitus related activity is unknown despite the fact that tinnitus usually displays a predominant pitch. The present study suggests that the neural network underlying tinnitus can be altered by almost any frequency. We could speculate that the inter-frequency connections of the central auditory system are aberrantly strong when in the presence of tinnitus and that any frequency is able to interact with the network in its whole. Another possibility (non-exclusive of the previous one) could be that tinnitus is underlain by the activity of neurons that are not selective in frequency. In other words, by neurons that can integrate auditory information originating from a large band of frequencies and may be located in the extra lemniscal system (Fournier et al., 2019; Møller, 2007; Møller et al., 1992).

Moreover, if we take into account the protocol used on the control group, and likewise the tinnitus subgroup (ST-CG and ST-TG), we modeled what may be considered as a tone-like peripheral tinnitus. Indeed, by presenting a continuous pure tone with similar characteristics to tinnitus (pitch and loudness), we were able to produce an ongoing peripheral neural activity (a ringing) that lasted during the whole session of testing. One may consider this activity to be similar to peripheral tinnitus-related activity, as the information is triggered in the periphery, and maintained in the auditory centers, as would be expected of peripheral tinnitus-related activity. When our participants were set in these configurations, the target was nearly impossible to suppress, and if so, the level required to generate the suppression was twice the level needed to suppress a real tinnitus sensation in these same participants. If these assumptions are validated, the prevalence of residual inhibition failure may represent the prevalence of peripheral-like tinnitus subtype which was reported to be around 15-30\% of all tinnitus cases (Fournier et al., 2018; Roberts et al., 2008; Vernon and Meikle, 1981). If this is true, RI may be the key element needed to distinguish tinnitus subtypes in patients, as the presence of RI would suggest central tinnitus, and the absence of RI would suggest peripheral tinnitus. In the sample tested here, all the tinnitus participants exhibited residual inhibition in multiple frequency masker conditions suggesting that they may all have central tinnitus subtype. The prevalence of MRIL in a larger tinnitus cohort using a similar methodology should definitely be attempted. 


\subsubsection{Duration of the suppression}

The main obvious difference between residual inhibition and forward masking have been previously noted by Feldmann (1984), he wrote: "It turns out that, in masking of tinnitus, there is often a considerable period of forward masking, in some cases 100 times as long as the corresponding effect in the stimulus versus-stimulus situation [when an external sound is the target]. It is called residual inhibition of tinnitus." He concluded that: "In masking of tinnitus the masking effect often extends considerably beyond the duration of the masking stimulus." His assumptions and observations were confirmed as many studies showed long-lasting effects of RI ranging from a few seconds to hours and, in the rarest cases, days after the cessation of a brief loud acoustic stimulation (Fournier et al., 2018; Hazell and Wood, 1981; Vernon and Meikle, 1981). In contrast, from what is known of forward masking, no experimental conditions have ever found a forward masking duration exceeding a delay of more than $200 \mathrm{~ms}$ (Moore, 2012). We can speculate that external sounds can deeply modify the neural activity flowing through the entire tinnitus-related network and that the tinnitus-related activity within the network may take some time to recover. In the case of an external sound, there is a constant physical source of energy (or "force") that is provided to the auditory system which then accounts for the very limited duration of forward masking. For tinnitus, on the other hand, tinnitus-related activity is internally generated via complex mechanisms involving, among others, balance between excitation and inhibition and connectivity (Eggermont and Tass, 2015; Noreña, 2011). Therefore, once the network has been perturbed (by an external sound, for instance), without any "driving force" it may require some time for the network to regain his initial (tinnitus) state with the associated tinnitus-related activity. The mechanisms accounting for RI duration are still unclear. If tinnitus is caused by hypersynchrony (Eggermont and Tass, 2015; Noreña and Farley, 2013; Roberts, 2007), it is possible that the higher the connectivity is within the tinnitus network the smaller the duration of RI may be. Therefore, a sound stimulation specifically designed to reduce the synchrony might increase RI duration. Moreover, such stimulation has been advocated and used as a sound therapy for tinnitus (Adamchic et al., 2017; Tass et al., 2012, 2019). If, on the other hand, tinnitus results from hyperactivity, RI duration may depend on the duration it takes for spontaneous activity to be restored at the tinnitus (hyperactive) level after a prolonged exposure to sound has decreased it. One notes that the two mechanisms are not exclusive from each other.

Overall, the differences in frequency selectivity and duration between forward masking of an external sound and residual inhibition of tinnitus suggests that these two phenomena are supported by different physiological mechanisms. It is important to note that they still share some common properties. For example, in both cases, the duration of the suppression expands with the increase of the masker level: the rate of recovery of forward masking increases as the intensity of the masker increases (Moore and Glasberg, 1983) and RI is proportional to the masker intensity (e.g., the recovery time of the tinnitus increases with increasing masker level) 
(Terry et al., 1983). Still, we found here that the level to trigger residual inhibition was far lower than the one required to trigger forward masking. They are also both affected by the duration of the masker: the amount of forward masking increases with increasing masker duration (Kidd and Feth, 1982; Zwicker, 1984) and residual inhibition duration increases linearly with the log of the masker duration (Terry et al., 1983).

Interestingly, the participants also reported a totally different experience for residual inhibition than for forward masking. Indeed, many controls and tinnitus participants reported a short delay in the detection of the simulated tinnitus signal in the silent interval for forward masking. The delay extended as the intensity of the masker increased; the appearance of the target sound was shifted more and more with the increase in intensity of the masker. This continued until the signal was delayed to the point where the controls could no longer hear the signal in the silent interval, or only a very brief "beep" before the next ramp of the sequence. In contrast, none of the tinnitus participants reported such perception when their real tinnitus was the target. In fact, some of them reported a gradual decrease of their tinnitus intensity as the intensity of the masker increased, or at least a certain "faintness" of their percept after the cessation of the acoustic stimulation. In itself, this shows how forward masking and residual inhibition are experienced in a very different fashion: the former is a delay or shift in the appearance of a fixed intensity target tone, a delay that expands with the increase of the masker level and the latter is a decrease in tinnitus intensity with the increase of the masker level.

\subsection{No phase configurations effect: diotic vs dichotic}

A secondary objective of the present research was to explore the effect of the phase configuration of the masker on the MML and the MRIL for both groups and both targets: the tinnitus and the simulated tinnitus. This was done with the goal of distinguishing between different tinnitus subtypes: for peripheral tinnitus subtype a MML difference would be expected while, for central tinnitus, no effect would be expected. Overall, across all the maskers tested here, no phase configuration effect was found for masking and poststimulation suppression for both groups. For tinnitus as the target, we can only compare the present MML results with previous ones, as there are, to our knowledge, no reports of the effect of phase configurations on residual inhibition. For MMLs, the current results are surprising given the previous studies on diotic and dichotic masking of tinnitus (Johnson and Hughes, 1992; Searchfield et al., 2016; Tyler and Stouffer, 1991) and emphasizes on the variability of tinnitus sensations across individuals. It is, however, difficult to compare our results to these studies, given the fact that no statistical analysis was conducted in these earlier reports. Still, according to the study conducted by Johnson and Hughes (1992), we should have observed lower MMLs in the dichotic configurations of our procedure. In fact, this observation led them to suggest that tinnitus could emerge from the auditory periphery. An explanation for the discrepancy between their results and ours could reside in the difference between the stimuli: they used 
large broadband noise (bandwidth of $>9000 \mathrm{~Hz}$ ) while we used narrowband noises ( 1 octave bandwidth). Even so, our large broadband noise condition (e.g. the white noise condition) did not show diotic and dichotic differences at group level. Furthermore, their masking level differences (MLD) were maintained with increasing center masker frequency whereas our absence of effect was conserved across all masker frequencies tested (Johnson and Hughes, 1992). On the other hand, the study by Tyler and Stouffer (1991) showed more variable results, but the time-delayed condition in their study had little effect for a majority of participants (less than $5 \mathrm{~dB}$ difference). It seems our results comfort the conclusion given by this study.

It is important to note that most participants in the present study reported a marked preference for the dichotic masker. Whether these reports are influenced by the detailed description of the study beforehand to all the participants is unknown, but a similar reaction was reported in the clinical trial conducted by Searchfield et al. (2016). Furthermore, the change in the phase of the stimuli was clearly recognized by a number of participants during the experiment for two frequency masker conditions: the reference frequency $(1 \mathrm{kHz})$ and the white noise. A common particularity of these two maskers is the presence of energy residing in the low frequency spectrum. This could be due to the fact that our auditory system uses inter-aural time differences (ITDs) more efficiently for sounds in the lower frequency spectrum (Moore, 2012), or because greater effects of BMLD have been associated with lower frequency ranges, below $1500 \mathrm{~Hz}$ (Colburn and Durlach, 1978). However, despite the reports given by the participants of a clear distinction made between diotic and dichotic phase configurations for these maskers, we did not observe greater effects of the phase on MML and MRIL for these two maskers. One potential explanation for this lack of effect could be that the frequency of the target signal to be masked and suppressed was in fact exceeding the limit for BMLD as described by Colburn and Durlach (1978). This is the most likely explanation since it has been shown that forward masking BMLD usually occurs only for low-frequency signals (Buss and Hall III, 2011; Yama, 1992; Yost and Walton, 1977). For both groups and both conditions, the target was always $\geq 4 \mathrm{kHz}$. For the control group, the target was always a $4 \mathrm{kHz}$ tone and for the tinnitus group, no participants had a pitch matching value lower than $4 \mathrm{kHz}$. We can thus speculate that for the external sound target condition, the frequency of the target was too high for BMLD to have an effect on the level of forward masking in the task. One can speculate that the absence of effect when the tinnitus was the target is due to similar reasons. Still, it could be interesting to replicate this study with tinnitus participants displaying a lower tinnitus frequency as assessed by pitch matching and a lower simulated tinnitus $(\leq 1000 \mathrm{~Hz})$ for the control group. Various reports of lower tinnitus pitch have been found in patients who suffer from Meniere's disease (Nodar and Graham, 1965; Perez-Carpena et al., 2019; Vernon et al., 1980). They may be ideal candidates for this task. On the three studies who have explored the effect of phase configurations of the maskers on MML when tinnitus is the target, two have not measured and/or reported the tinnitus pitch of their participants (Johnson and Hughes, 1992; Tyler and Stouffer, 1991) and one tested tinnitus participants with only high frequency tinnitus (Searchfield et al., 2016). 


\subsection{Differences between the present study and that of Fournier et al. (2018)}

The ramped sequence used here differentiated itself from the one used in the study of Fournier et al. (2018) in different ways: (1) the rise and fall time of our sequence were fixed at 1.5 and $2 \mathrm{~s}$, respectively (2) the looped sequence was made so that the intensity was ever changing throughout the time course of the loop and (3) the peak time as well as the silence time (when the intensity falls back to 0 ) was only a fraction of a second. This was done in order to deliver a more comfortable stimulation to the ears of the end users. This also means that the sequence is always stimulating the listeners' ears and that the silent gap is only apparent due to infra-threshold activity. This pseudo-continuous stimulation might potentiate the inhibition of tinnitus: this might explain why all tinnitus participants achieved residual inhibition in at least one condition of the present study (100\%) in comparison to a lower prevalence in previous ones (87\% for Fournier et al., 2018; 75\% for Vernon and Meikle, 1981; $66 \%$ for Roberts et al., 2008). In addition, this type of stimulation might also have been optimal to measure forward masking as the time delay generated by this phenomenon rarely exceeds $200 \mathrm{~ms}$ (Moore, 2012) and that the apparent silent gap was only a fraction of a second. In addition, as the level of the masker increased, the slopes of the rise and fall times increased leading to an "apparent gap" duration decrease: some of the infra-threshold activity should have become suprathreshold activity with the increase in masker level thus reducing the apparent duration of the silent gap. This could explain why forward masking was present only at high intensity levels for the external sound target: as the gap was reduced with increasing levels of the masker and, coincidentally, the forward masking duration was also increased by the increase of the masker level, the disappearance of the target tone happened only at the masker level where the duration of the delay was equal to the duration of the silent gap. Using the original method developed by Fournier et al. (2018) with pulse noise of short rise and fall times and a silent gap duration of $1 \mathrm{~s}$ would probably have resulted in the impossibility of measuring any suppression of an external sound target during the gap as the duration of the silent gap would have exceeded the maximum forward masking time delay of $200 \mathrm{~ms}$. The original technique may be thus more suited to differentially diagnose different tinnitus subtypes but conversely be less suited to measure forward masking.

\section{Conclusion}

In conclusion, by using a novel method designed to measure the minimum level of residual inhibition (MRIL), the current study showed that poststimulation suppression of tinnitus is clearly different from poststimulation suppression of an external sound with similar characteristics (pitch and loudness). When tinnitus was the target, there was a high proportion of achievable MRIL for almost every masker frequency. Conversely, when an external sound was the target, MRIL was 
achievable only for the masker frequency that encompassed the frequency of the external sound target. These results suggest that all the tinnitus participants of the current study displayed central tinnitus subtype. We believe that MRIL measurements should be used routinely as a diagnostic test for tinnitus in clinics. As previously stated (Fournier et al., 2018), this technique is faster than the previous classical method for measuring residual inhibition (Vernon, 1977) and could possibly discriminate between peripheral and central tinnitus subtypes. The diagnostic value of MRIL should be tested on larger cohorts of tinnitus patients with different etiologies.

\section{References}

Adamchic, I., Toth, T., Hauptmann, C., Walger, M., Langguth, B., Klingmann, I., Tass, P.A., 2017. Acute effects and after-effects of acoustic coordinated reset neuromodulation in patients with chronic subjective tinnitus. Neuroimage Clin. 15, 541-558.

Baguley, D., McFerran, D., Hall, D., 2013. Tinnitus. Lancet 382, 1600-1607.

Basile, C.-É., Fournier, P., Hutchins, S., Hébert, S., 2013. Psychoacoustic assessment to improve tinnitus diagnosis. PLoS One 8, e82995.

Burns, E.M., 1984. A comparison of variability among measurements of subjective tinnitus and objective stimuli. Int. J. Audiol. 23, 426-440.

Buss, E., Hall III, J.W., 2011. Effects of non-simultaneous masking on the binaural masking level difference. J. Acoust. Soc. Am. 129, 907-919.

Cazals, Y., Dauman, R., 1990. Tinnitus deterioriates frequency selectivity in addition to hearing loss. In: Kemp, D.T., Cianfrone, G., Grandori, F. (Eds.), Cochlear Mechanisms and Otoacoustic Emissions, Advances in Audiology. S. Karger, pp. 201-205.

Cederroth, C.R., Gallus, S., Hall, D.A., Kleinjung, T., Langguth, B., Maruotti, A., Meyer, M., Norena, A., Probst, T., Pryss, R., Searchfield, G., Shekhawat, G., Spiliopoulou, M., Vanneste, S., Schlee, W., 2019. Editorial: towards an understanding of tinnitus heterogeneity. Front. Aging Neurosci. 11, 1-7.

Colburn, H.S., Durlach, N.I., 1978. Models of binaural interaction. Handbook of Perception. 4, 467-518.

Dauman, R., Cazals, Y., 1989. Auditory frequency selectivity and tinnitus. Arch. Otorhinolaryngol. 246, 252-255.

Eggermont, J.J., Roberts, L.E., 2004. The neuroscience of tinnitus. Trends Neurosci. $27,676-682$.

Eggermont, J.J., Tass, P.A., 2015. Maladaptive neural synchrony in tinnitus: origin and restoration. Front. Neurol. 6, 29.

Feldmann, H., 1971. Homolateral and contralateral masking of tinnitus by noise-bands and by pure tones. Audiology 10, 138-144.

Feldmann, H., 1983. Time patterns and related parameters in masking of tinnitus. Acta Otolaryngol. 95, 594-598.

Feldmann, H., 1984. Masking-mechanisms (IPSI, contralateral masking). J. Laryngol. Otol. 98, 54-58.

Fournier, P., Cuvillier, A.-F., Gallego, S., Paolino, F., Paolino, M., Quemar, A., Londero, A., Norena, A., 2018. A new method for assessing masking and residual inhibition of tinnitus. Trends Hear. 22, 233121651876999. 
Fournier, P., Wrzosek, M., Paolino, M., Paolino, F., Quemar, A., Noreña, A.J., 2019. Comparing tinnitus tuning curves and psychoacoustic tuning curves. Trends Hear. 23, 233121651987853.

Fowler, E.P., 1940. Head noises: significance, measurement and importance in diagnosis and treatment. Arch. Otolaryngol. Head Neck Surg. 32, 903-914.

Fowler, E.P., 1941. XII tinnitus aurium in the light of recent research. Ann. Otol. Rhinol. Laryngol. 32, 903-914

Galazyuk, A.V., Voytenko, S.V., Longenecker, R.J., 2017. Long-lasting forward suppression of spontaneous firing in auditory neurons: implication to the residual inhibition of tinnitus. J. Assoc. Res. Otolaryngol. 18, 343-353.

Goodwin, P.E., Johnson, R.M., 1980. The loudness of tinnitus. Acta Otolaryngol. 90, 353-359.

Guitton, M., Dudai, Y., 2007. Blockade of cochlear NMDA receptors prevents long-term tinnitus during a brief consolidation window after acoustic trauma. Neural Plast. 2007, 80904.

Guitton, M., Puel, J., 2004. Cochlear NMDA receptors and tinnitus. Audiolog. Med. 2, 3-7.

Hallam, R.S., Jakes, S.C., Chambers, C., Hinchcliffe, R., 1985. A comparison of different methods for assessing the "intensity" of tinnitus. Acta Otolaryngol. 99, 501-508.

Harrell, R.W., 2002. Puretone evaluation. In: Katz, J. (Ed.), Handbook of Clinical Audiology. Lippincott Williams \& Wilkins, Baltimore, pp. 71-87.

Harris, D.M., Dallos, P., 1979. Forward masking of auditory nerve fiber responses. J. Neurophysiol. 42, 1083-1107.

Hawkins, D.B., Wightman, F.L., 1980. Interaural time discrimination ability of listeners with sensorineural hearing loss. Audiology 19, 495-507.

Hazell, J.W., Wood, S., 1981. Tinnitus masking-a significant contribution to tinnitus management. Br. J. Audiol. 15, 223-230.

Hébert, S., 2018. Individual reliability of the standard clinical method vs patient-centered tinnitus likeness rating for assessment of tinnitus pitch and loudness matching. JAMA Otolaryngol. Head Neck Surg. 144, 1136-1144.

Hébert, S., Fournier, P., 2017. Clinical validation of a new tinnitus assessment technology. Front. Neurol. 8, 1-8.

Jerger, J., Brown, D., Smith, S., 1984. Effect of peripheral hearing loss on the masking level difference. Arch. Otolaryngol. 110, 290-296.

Johnson, R.M., Hughes, F.M., 1992. Diotic Versus Dichotic Masking of Tinnitus, Presented at the Fourth International Tinnitus Seminar, Kugler, Bordeaux.

Kidd, G., Feth, L.L., 1982. Effects of masker duration in pure tone forward masking. J. Acoust. Soc. Am. 72, 1384-1386.

Kluk, K., Moore, B.C.J., 2006. Detecting dead regions using psychophysical tuning curves: A comparison of simultaneous and forward masking: La detección de regiones muertas utilizando curvas psicofísicas de afinamiento: Una comparación del enmascaramiento simultáneo y el anterógrado. Int. J. Audiol. 45, 463-476.

Landgrebe, M., Zeman, F., Koller, M., Eberl, Y., Mohr, M., Reiter, J., Staudinger, S., Hajak, G., Langguth, B., 2010. The tinnitus research initiative (TRI) database: a new approach for delineation of tinnitus subtypes and generation of predictors for treatment outcome. BMC Med. Inform. Decis. Mak. 10, 1-7.

Langenbeck, B., 1953. Diagnosis by testing hearing above threshold. Acta Otolaryngol. 43, 439-456.

McCormack, A., Edmondson-Jones, M., Fortnum, H., Dawes, P., Middleton, H., Munro, K.J., Moore, D.R., 2014. The prevalence of tinnitus and the relationship with neuroticism in a middle-aged UK population. J. Psychosom. Res. 76, 56-60. 
McCormack, A., Edmondson-Jones, M., Somerset, S., Hall, D., 2016. A systematic review of the reporting of tinnitus prevalence and severity. Hear. Res. 337, 70-79.

McFerran, D.J., Stockdale, D., Holme, R., Large, C.H., Baguley, D.M., 2019. Why is there no cure for tinnitus? Front. Neurosci. 13, 1-13.

Mckenna, L., 2004. Models of tinnitus suffering and treatment compared and contrasted. Audiol. Med. 2, 41-53.

Meddis, R., O'Mard, L.P., 2005. A computer model of the auditory-nerve response to forwardmasking stimuli. J. Acoust. Soc. Am. 117, 3787-3798.

Mitchell, C., 1983. The masking of tinnitus with pure tones. Int. J. Audiol. 22, 73-87.

Mitchell, C., Vernon, J., Creedon, T., 1993. Measuring tinnitus parameters: loudness, pitch, and maskability. J. Am. Acad. Audiol. 4, 139-151.

Moffat, G., Adjout, K., Gallego, S., Thai-Van, H., Collet, L., Noreña, A.J., 2009. Effects of hearing aid fitting on the perceptual characteristics of tinnitus. Hear. Res. 254, 82-91.

Møller, A.R., 2007. The role of neural plasticity in tinnitus. Prog. Brain Res. 166, 37-45.

Møller, A.R., Møller, M.B., Yokota, M., 1992. Some forms of tinnitus may involve the extralemniscal auditory pathway. Laryngoscope 102, 1165-1171.

Moore, B.C.J., 2012. Frequency selectivity, masking, and the critical band. In: An Introduction to the Psychology of Hearing. Emerald Group Publishing Limited, Bingley, UK, pp. 67-131.

Moore, B.C., Alcántara, J.I., 2001. The use of psychophysical tuning curves to explore dead regions in the cochlea. Ear Hear. 22, 268-278.

Moore, B.C., Glasberg, B.R., 1983. Growth of forward masking for sinusoidal and noise maskers as a function of signal delay; implications for suppression in noise. J. Acoust. Soc. Am. 73, 1249-1259.

Nodar, R.H., Graham, J.T., 1965. An investigation of frequency characteristics of tinnitus associated with Meniere's disease. Arch. Otolaryngol. 82, 28-31.

Noreña, A.J., 2011. An integrative model of tinnitus based on a central gain controlling neural sensitivity. Neurosci. Biobehav. Rev. 35, 1089-1109.

Noreña, A.J., Farley, B.J., 2013. Tinnitus-related neural activity: theories of generation, propagation, and centralization. Hear. Res. 295, 161-171.

Norena, A., Micheyl, C., Chéry-Croze, S., Collet, L., 2002. Psychoacoustic characterization of the tinnitus spectrum: implications for the underlying mechanisms of tinnitus. Audiol. Neurootol. 7, 358-369.

Oxenham, A.J., 2001. Forward masking: adaptation or integration? J. Acoust. Soc. Am. 109, 732-741.

Oxenham, A.J., Moore, B.C., 1994. Modeling the additivity of nonsimultaneous masking. Hear. Res. 80, 105-118.

Oxenham, A.J., Plack, C.J., 2000. Effects of masker frequency and duration in forward masking: further evidence for the influence of peripheral nonlinearity. Hear. Res. 150, 258-266.

Penner, M.J., Brauth, S., Hood, L., 1981. The temporal course of the masking of tinnitus as a basis for inferring its origin. J. Speech Hear. Res. 24, 257-261.

Perez-Carpena, P., Martinez-Martinez, M., Martínez Carranza, R.A., Batuecas-Caletrio, A., Lopez-Escamez, J.A., 2019. A tinnitus symphony in 100 patients with Meniere's disease. Clin. Otolaryngol. 44, 1176-1180.

Plack, C.J., Oxenham, A.J., Drga, V., 2002. Linear and nonlinear processes in temporal masking. Acta Acust. United AC 88, 11.

Roberts, L.E., 2007. Residual inhibition. In: Progress in Brain Research. Elsevier, pp. 487-495. 


\section{Difference between residual inhibition and forward masking}

Roberts, L.E., Salvi, R., 2019. Overview: hearing loss, tinnitus, hyperacusis, and the role of central gain. Neuroscience 407, 1-7.

Roberts, L.E., Moffat, G., Bosnyak, D.J., 2006. Residual inhibition functions in relation to tinnitus spectra and auditory threshold shift. Acta Otolaryngol. Suppl. 27-33.

Roberts, L.E., Moffat, G., Baumann, M., Ward, L.M., Bosnyak, D.J., 2008. Residual inhibition functions overlap tinnitus spectra and the region of auditory threshold shift. J. Assoc. Res. Otolaryngol. 9, 417-435.

Schaette, R., 2014. Tinnitus in men, mice (as well as other rodents), and machines. Hear. Res. $311,63-71$.

Schaette, R., McAlpine, D., 2011. Tinnitus with a normal audiogram: physiological evidence for hidden hearing loss and computational model. J. Neurosci. 31, 13452-13457.

Schecklmann, M., Vielsmeier, V., Steffens, T., Landgrebe, M., Langguth, B., Kleinjung, T., 2012. Relationship between audiometric slope and tinnitus pitch in tinnitus patients: insights into the mechanisms of tinnitus generation. PLoS One 7, 1-7.

Searchfield, G.D., Kobayashi, K., Hodgson, S.-A., Hodgson, C., Tevoitdale, H., Irving, S., 2016. Spatial masking: development and testing of a new tinnitus assistive technology. Assist. Technol. 28, 115-125.

Sereda, M., Hall, D.A., Bosnyak, D.J., Edmondson-Jones, M., Roberts, L.E., Adjamian, P., Palmer, A.R., 2011. Re-examining the relationship between audiometric profile and tinnitus pitch. Int. J. Audiol. 50, 303-312.

Shore, S.E., Roberts, L.E., Langguth, B., 2016. Maladaptive plasticity in tinnitus-triggers, mechanisms and treatment. Nat. Rev. Neurol. 12, 150-160.

Stouffer, J.L., Tyler, R.S., 1990. Characterization of tinnitus by tinnitus patients. J. Speech Hear. Disord. 55, 439-453.

Tass, P.A., Adamchic, I., Freund, H.-J., von Stackelberg, T., Hauptmann, C., 2012. Counteracting tinnitus by acoustic coordinated reset neuromodulation. Restor. Neurol. Neurosci. 30, 137-159.

Tass, P.A., Silchenko, A.N., Popelka, G.R., 2019. Acoustic coordinated reset therapy for tinnitus with perceptually relevant frequency spacing and levels. Sci. Rep. 9, 13607.

Terry, A.M., Jones, D.M., Davis, B.R., Slater, R., 1983. Parametric studies of tinnitus masking and residual inhibition. Br. J. Audiol. 17, 245-256.

Tyler, R.S., Conrad-Armes, D., 1984. Masking of tinnitus compared to masking of pure tones. J. Speech Hear. Res. 27, 106-111.

Tyler, R.S., Stouffer, J.L., 1991. Binaural Tinnitus Masking With a White Noise Centered on the Tinnitus, Presented at the fourth International Tinnitus Seminar, Kugler, Bordeaux, pp. 391-394.

Vernon, J., 1977. Attemps to relieve tinnitus. J. Am. Audiol. Soc. 2, 8.

Vernon, J., Meikle, M., 1981. Tinnitus masking: unresolved problems. Ciba Found. Symp. $85,239-262$.

Vernon, J., Johnson, R., Schleuning, A., 1980. The characteristics and natural history of tinnitus in Meniere's disease. Otolaryngol. Clin. North Am. 13, 611-619.

Yama, M.F., 1992. Effects of temporal separation and masker level on binaural analysis in forward masking. J. Acoust. Soc. Am. 91, 327-335.

Yost, W.A., Walton, J., 1977. Hierarchy of masking-level differences obtained for temporal masking. J. Acoust. Soc. Am. 61, 1376-1379.

Zwicker, E., 1984. Dependence of post-masking on masker duration and its relation to temporal effects in loudness. J. Acoust. Soc. Am. 75, 219-223. 